\title{
Bosonic Josephson effect in the Fano-Anderson model
}

\author{
G. Engelhardt. ${ }^{*}$ G. Schaller, and T. Brandes \\ Institut für Theoretische Physik, Technische Universität Berlin, Hardenbergstr. 36, 10623 Berlin, Germany
}

\begin{abstract}
We investigate the coherent dynamics of a non-interacting Bose-Einstein condensate in a system consisting of two bosonic reservoirs coupled via a spatially localized mode. We describe this system by a two-terminal Fano-Anderson model and investigate analytically the time evolution of observables such as the bosonic Josephson current. In doing so, we find that the Josephson current sensitively depends on the on-site energy of the localized mode. This facilitates to use this setup as a transistor for a Bose-Einstein condensate. We identify two regimes. In one regime, the system exhibits well-behaved long-time dynamics with a slowly oscillating and undamped Josephson current. In a second regime, the Josephson current is a superposition of an extremely weakly damped slow oscillation and an undamped fast oscillation. Our results are confirmed by finite-size simulations.
\end{abstract}

PACS numbers: 05.60.Gg, 03.75.-b, 67.85.-d, 72.10.Bg

\section{INTRODUCTION}

The experimental control of cold-atomic quantum gases has proceeded to a high level in the recent years. In particular, transport experiments in two-terminal setups exhibit interesting effects as, e.g., conductance quantization or the creation of a heat engine $[1+3]$. Moreover, the control of superfluids gives rise to new transport regimes: in contrast to particle transport driven by a difference of the chemical potentials in the reservoirs or by a temperature gradient, the dynamics of a superfluid is determined by the phase of its matter wave [4] 6 . The control of these kinds of systems could pave the way to establish so-called atomtronic circuits [7 9].

Moreover, theoretical investigations of bosonic transport predict interesting effects as, e.g., a quantization of the current, superfluid Helmholtz oscillations or current against the chemical potential gradient [10 16. However, all of them rely to some extend on phenomenological assumptions to describe the many-particle dynamics, as interactions destroy integrability.

Motivated by these experimental and theoretical achievements, we study here the dynamics of a system which consists of two bosonic reservoirs which may at low temperature contain Bose-Einstein condensates (BECs). These reservoirs are linked by an additional strongly confined potential well. The situation is sketched in Fig. 1(a). For weak couplings and low temperature, the dynamics is mainly governed by a bosonic Josephson effect [17-19]. Thereby, the Josephson current between two directly coupled BECs depends on the phase difference of the condensates. Here we investigate, how the indirect coupling via the additional potential well influences the dynamics. Furthermore, a special focus of this article is the influence of the excited reservoir modes on the dynamics of the BEC. Based on our model and our methods, we are able to analytically investigate the effects of particle loss and damping due to these excited reservoir

\footnotetext{
* georg@itp.tu-berlin.de
}

modes.

In contrast to the theoretical investigations in Refs. [10 16, we maintain the integrability in our investigation by neglecting the inter-particle interactions. For instance, in Rubidium condensates, the interaction is rather small and can be additionally adjusted using Feshbach resonances [20, 21]. The absence of interactions allows us to analytically solve the dynamics of the full system. Here, the only additional assumption enters by presupposing the excited reservoir states to be thermally occupied initially. We describe this system as a Fano-Anderson model, which allows for analytical calculations. Our aim is to understand the effects in the non-interacting model in detail, which can then provide a starting point for future investigations of the behavior in the presence of interactions. In particular, we derive an effective non-hermitian Hamiltonian describing exactly the dynamics of the condensate in the long-time limit. Our methods could be thus employed to microscopically study effects which appear in non-hermitian Hamiltonians as, e.g., the so-called exceptional points, where two eigenvalues and their eigenstates merge as a function of system parameters 22 25.

An indispensable device in integrated electronic circuits is a transistor. By adjusting a gate potential, one can control the current from a source to a drain region with high accuracy. We investigate the dynamics of the BEC in a bosonic Fano-Anderson model in order to test if a bosonic system can be applied as a transistor-like device controlling the Josephson current. The confined potential well is assumed to have a large trapping frequency so that it is justified to consider it as single mode with energy $\epsilon$. For this reason, we call the confined potential well a 'bosonic quantum dot'. In the following, due to the close relation to a common transitor, we denote $\epsilon$ with the gate potential. We demonstrate that the Josephson current from the left to the right reservoir sensitively depends on $\epsilon$. Furthermore, our approach reveals two regimes in the dynamics induced by the excited reservoir modes. Depending on the gate potential, there is a regime with a constant dot occupation in the long-time limit, and a regime where the dot occupation exhibits 

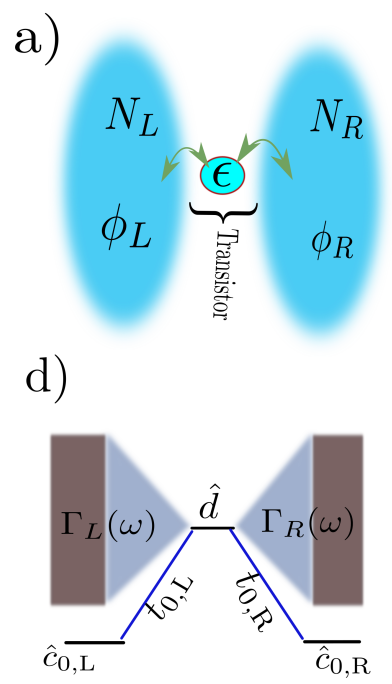

c)

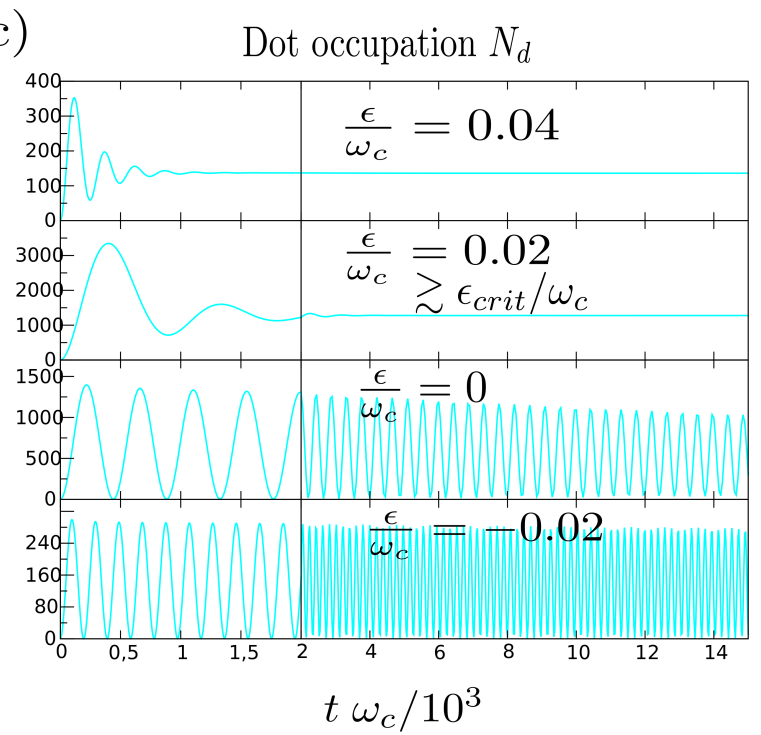

FIG. 1. (Color online) (a) Sketch of the system. Two reservoirs are coupled via a strongly confined well. (b) The system is described by the Fano-Anderson model in Eq. [6). (c) Dot occupation $N_{d}$ as function of time for different gate potentials $\epsilon$. For $\epsilon>\epsilon_{\text {crit }} \approx 0.016 \omega_{c}$, the occupation is constant, while it is oscillating for $\epsilon<\epsilon_{\text {crit }}$ in the long-time limit. (d) In our calculations, we formally exclude the coupling of the lowest energy modes $\mathbf{c}_{0, \alpha}$ in the reservoirs $\alpha=L, R$ from the continuum limit (CL) to take into account the dynamics of the BEC. (e) Dot occupation $N_{d}$ as function of $\epsilon$ for a fixed time $t_{1} \omega_{c}=2 \cdot 10^{3}$. The occupation is extremely high close to the transition at $\epsilon=\epsilon_{\text {crit }}$. The black line depicts the analytic time-averaged dot occupation in Eq. (52). The overall parameters are $n_{L}=n_{R}=10^{4}, \Delta \phi=-\pi / 2, \eta=0.5, \gamma_{\alpha} / \omega_{c}=0.028$, and $t_{0, \alpha} / \omega_{c}=0.0021$ and $\omega_{0, \alpha}=0$. For more details about the choice of $t_{0, \alpha}$ see appendix A.

fast oscillations which persists for very long times. At the transition, the dot occupation is exceedingly high. The Josephson current exhibits a similar behavior. The regimes appear as the energy of the reservoir modes is bounded at energy $\omega=0$. As the energy of the eigenstates of the system generating the dynamics is below or above that boundary, their dynamics is subjected to damping or not.

The structure of the article is as follows. In Sec. IIA. we give a general introduction to the Josephson effect in superconducting and bosonic systems. In Sec. IIB, we explain the bosonic Fano-Anderson model, for which we specify the Josephson current in Sec. IIIA In Sec. IIIB, we apply the so-called equations-of-motion method to calculate the dynamics, which is discussed in Sec. IIIC, Section IV focuses on the dynamics for long times. In Secs. IV A IV C, we explain how to efficiently calculate the time evolution and show how to derive an effective Hamiltonian resembling exactly the dynamics in the long-time limit. We provide an exact expression for the Josephson current in Sec. IV D. In Sec. IVE, we discuss the low-frequency current which is mainly responsible for the particle transport. The appendix provides details about the calculations.

\section{THE SYSTEM AND BASICS}

\section{A. The Josephson effect}

In a conventional superconductor, the Cooper-pairs form a condensate whose macroscopic order parameter is described by a phase $\phi$. Two superconducting regions connected by a small normal-conducting island constitute a Josephson junction. The coherent Josephson current through this junction depends on the phase difference $\Delta \phi=\phi_{R}-\phi_{L}$ of the condensate phases in the superconducting leads, namely

$$
I_{J}(t)=I_{c} \sin \Delta \phi(t)
$$

where $I_{c}$ is called the critical current [17, 18. In the acJosephson effect, the two leads are subjected to a chemical potential bias $\Delta \mu$ which gives rise to a time evolution of the phase difference

$$
\frac{d}{d t} \Delta \phi(t)=\frac{2 e}{\hbar} \Delta \mu
$$

where $2 e$ is the charge of a Cooper pair. For a constant bias $\Delta \mu$, this results in a sine-modulated Josephson current with the so-called characteristic frequency of the junction $\Omega_{J}=\frac{2 e}{\hbar} \Delta \mu$.

An analogue effect appears also in a BEC, whose macroscopic order parameter is the complex-valued wave function. The simplest model with a bosonic Josephson 
current consists of two coupled bosonic modes

$$
H=t_{0}\left(\mathbf{a}^{\dagger} \mathbf{b}+\mathbf{b}^{\dagger} \mathbf{a}\right)
$$

where $t_{0}$ denotes the tunneling coupling [19. The bosonic Josephson current related to the operator $\mathbf{I} \equiv i\left[H, \mathbf{b}^{\dagger} \mathbf{b}\right]$ reads

$$
I_{J}(t)=t_{0} 2 \operatorname{Im}\left\langle\mathbf{b}^{\dagger} \mathbf{a}\right\rangle_{t} .
$$

For the initial state $\left|\psi_{0}\right\rangle$ at time $t=0$ we assume a BEC described by a product of coherent states $\left|\psi_{0}\right\rangle=|a\rangle \otimes$ $|b\rangle$ with $\mathbf{a}|a\rangle=\sqrt{n_{a}} e^{-i \phi_{a}}|a\rangle$ and $\mathbf{b}|b\rangle=\sqrt{n_{b}} e^{-i \phi_{b}}|b\rangle$. Thereby, $n_{\alpha}$ denotes the initial occupation of mode $\alpha=$ $a, b$ and $\phi_{\alpha}$ its phase. Solving the equations of motion we find

$$
\begin{aligned}
I_{J}(t)= & -\frac{1}{2} \Omega_{J} \sin \left(\Omega_{J} t\right)\left(n_{b}-n_{a}\right) \\
& +\Omega_{J} \cos \left(\Omega_{J} t\right) \sin \Delta \phi_{0} \sqrt{n_{a} n_{b}},
\end{aligned}
$$

where $\Delta \phi_{0}=\phi_{b}-\phi_{a}$ and $\Omega_{J}=2 t_{0}$ is the characteristic frequency. In contrast to (2), we do not assume a difference in the chemical potentials in the bosonic Hamiltonian as finite energy terms $\omega_{a} \mathbf{a}^{\dagger} \mathbf{a}$ and $\omega_{b} \mathbf{b}^{\dagger} \mathbf{b}$ are not present in Eq. (3). Obviously, the Josephson current exhibits an oscillating behavior with the characteristic frequency $\Omega_{J}=2 t_{0}$. This is exactly the energy difference of the two eigenenergies $\epsilon_{ \pm}= \pm t_{0}$ of the Hamiltonian (3). The superposition of the corresponding eigenmodes thus drives the coherent dynamics of the Josephson current. The same effect also generates the BEC dynamics in the Fano-Anderson model considered in this article, although here the coherent dynamics is subjected to incoherent particle loss due to the coupling to the excited reservoir modes. In Sec. IVE we derive a relation similar to (5) for the low-frequency Josephson current in the FanoAnderson model, c.f. Eq. (49). We find, that the excited reservoir modes effectively create an additional imaginary part to the energies and to the characteristic frequency $\Omega_{J}$.

\section{B. Our model}

We theoretically model the transport system depicted in Fig. 1 (a) as a bosonic two-terminal Fano-Anderson model to investigate the transport properties of a BEC which, to our knowledge, has not been done before. The model is sketched in Fig. 1(b). The Hamiltonian reads

$$
\begin{aligned}
& \mathbf{H}=\epsilon \mathbf{d}^{\dagger} \mathbf{d}+\sum_{\alpha=L, R} \sum_{k=0}^{k_{\max }} {\left[\omega_{k \alpha} \mathbf{c}_{k \alpha}^{\dagger} \mathbf{c}_{k \alpha}\right.} \\
&\left.+\left(t_{k \alpha} \mathbf{d}^{\dagger} \mathbf{c}_{k \alpha}+\text { h.c. }\right)\right],
\end{aligned}
$$

where $\omega_{k \alpha}$ denote the energy of the bosonic reservoir modes $\mathbf{c}_{k \alpha}$ which are labeled by $\alpha=L, R$ denoting the reservoirs and $k=0, \ldots, k_{\max }$ denoting their internal states. The parameters $t_{k \alpha}$ describe the coupling of the dot $\mathbf{d}$ to each reservoir mode. Without loss of generality, we assume real-valued $t_{k \alpha}$ throughout the article. Complex tunneling elements can be rendered real by an appropriate gauge transformation. This and related models have been frequently used to study transport in various contexts [26 31]. For a bosonic Fano-Anderson model at temperatures above the condensation temperature, one can show that the stationary particle current is given by 32

$$
I_{R}=\int_{0}^{\infty} \mathcal{G}(\omega)\left[n_{L}(\omega)-n_{R}(\omega)\right] d \omega,
$$

where $n_{\alpha}(\omega)=1 /\left[e^{\beta_{\alpha}\left(\omega-\mu_{\alpha}\right)}-1\right]$ is the Bose distribution and describes the occupation of the left and right reservoir modes. It depends on the chemical potentials $\mu_{\alpha}<0$ and the temperatures $T_{\alpha}=1 / \beta_{\alpha}$. The transmission $\mathcal{G}(\omega)$ is a system property and does not depend on either temperature or chemical potential. Thus, the current through the system is generated by a difference of the chemical potentials or temperatures in the reservoirs. In contrast, we are interested in the coherent contributions to the particle transfer which - just as in the bosonic two-mode system of Sec. II A - can be present even in the case of vanishing temperature and chemical potential difference.

\section{DYNAMCIS OF THE BOSE-EINSTEIN CONDENSATE}

\section{A. Josephson current in the Fano-Anderson model}

We define the current operator via the time evolution of the particle-number operator of the right reservoir $\mathbf{N}_{R}=\sum_{k} \mathbf{c}_{k, R}^{\dagger} \mathbf{c}_{k, R}$, thus,

$$
\begin{aligned}
\mathbf{I}_{R} & \equiv i\left[\mathbf{H}, \mathbf{N}_{R}\right]=-i \sum_{k} t_{k, R} \mathbf{c}_{k, R}^{\dagger} \mathbf{d}+\text { h.c. } \\
& \equiv \mathbf{I}_{R, e x}+\mathbf{I}_{R, J} .
\end{aligned}
$$

The current can be split into two parts $\mathbf{I}_{R, e x}$ and $\mathbf{I}_{R, J}$. They are related to the initial condition which we specify in the following.

The density matrix at time $t=0$ is given by a product of the density matrices describing each reservoir separately. The excited states are assumed to be initially thermally occupied. Moreover, we allow for a condensate in each reservoir $\alpha$ so that the lowest energy modes $\omega_{0, \alpha}$ are macroscopically occupied. This effect requires a finite energy gap between the lowest energy mode and the excited modes. If the temperature of the reservoir is lower than a critical temperature which depends on the particle density, the Bose-Einstein condensation takes place.

Each condensate in the modes $\mathbf{c}_{0, \alpha}$ is characterized by a macroscopic occupation $n_{\alpha}$ and a phase $\phi_{\alpha}$. We describe it by a coherent state $\left|\alpha_{0}\right\rangle$. For these reasons, the 
initial density matrix reads

$$
\begin{aligned}
\rho & =\rho_{L} \otimes \rho_{R} \otimes \rho_{d} \\
\rho_{\alpha} & =\frac{1}{Z_{\alpha}} \exp \left[-\beta_{\alpha} \sum_{k \neq 0}^{k_{\max }} \omega_{k, \alpha} \mathbf{c}_{k, \alpha}^{\dagger} \mathbf{c}_{k, \alpha}\right] \otimes\left|\alpha_{0}\right\rangle\left\langle\alpha_{0}\right|
\end{aligned}
$$$$
\begin{aligned}
\mathbf{c}_{0 \alpha}\left|\alpha_{0}\right\rangle & =\alpha_{0}\left|\alpha_{0}\right\rangle \quad \text { with } \quad\left\langle\alpha_{0} \mid \alpha_{0}\right\rangle=1, \\
\rho_{d} & =|0\rangle\langle 0|,
\end{aligned}
$$

where $\alpha_{0}=\sqrt{n_{\alpha}} e^{-i \phi_{\alpha}}, \beta_{\alpha}$ denotes the inverse temperature, and $|0\rangle$ is the vacuum state of the dot. The excited modes are described by a density matrix of a grandcanonical ensemble. As we consider a BEC, we assume vanishing chemical potentials $\mu_{\alpha} \rightarrow 0$. The partition function $Z_{\alpha}$ warrants the normalization $\operatorname{tr} \rho_{\alpha}=1$. As the experiments in Refs. 4, 33] demonstrate, the initial ground-state occupations $n_{\alpha}$ and the phases $\phi_{\alpha}$ can be controlled with high accuracy.

Accordingly, we split the current into two contributions. The first one is the current operator from and to the excited states $k>0$, thus

$$
\mathbf{I}_{R, e x}=-i \sum_{k \neq 0} t_{k, R} \mathbf{c}_{k, R}^{\dagger} \mathbf{d}+\text { h.c. }
$$

Particles which are thermally excited at $t=0$ generate a current given by Eq. (7) in the long-time limit which is the main part of $\mathbf{I}_{R, e x}$. Furthermore, particles which are initially in the condensate do not necessarily stay there. During the time evolution they can jump into the dot and then into an excited mode. Thus, also a fraction of the condensate particles can participate in $\mathbf{I}_{R, e x}$.

In the presence of a condensate we identify the current from and to the reservoir ground states $k=0$ as the Josephson current which is coherent. The corresponding current operator reads

$$
\mathbf{I}_{R, J}=-i t_{0, R} \mathbf{c}_{0, R}^{\dagger} \mathbf{d}+\text { h.c. } .
$$

In this article, we are interested in the latter contribution. We therefore assume the zero-temperature limit $T_{\alpha} \rightarrow 0$, or equivalently $\beta_{\alpha} \rightarrow \infty$, where all particles are initially condensed within the lowest energy modes $\mathbf{c}_{0 \alpha}$.

\section{B. Equations of motion in Laplace space}

Following Ref. 32, we construct the Heisenberg equations of motion $\frac{d}{d t} \mathbf{o}=i[\mathbf{H}, \mathbf{o}]$ for $\mathbf{o}=\mathbf{d}, \mathbf{c}_{k \alpha}$ and apply a Laplace transformation $\hat{\mathbf{o}}(z) \equiv \int_{0}^{\infty} e^{-z t} \mathbf{o}(t) d t$. In Laplace space, the equations of motions can be easily solved and we obtain

$$
\begin{aligned}
\hat{\mathbf{d}}(z) & =\frac{d}{\mathcal{F}(z)}-i \sum_{\alpha=L, R} \sum_{k=0}^{k_{\max }} \frac{t_{k, \alpha} c_{k, \alpha}}{\left(z+i \omega_{k, \alpha}\right) \mathcal{F}(z)}, \\
\hat{\mathbf{c}}_{k, \alpha}(z) & =\frac{c_{k, \alpha}}{z+i \omega_{k, \alpha}}-\frac{i t_{k, \alpha} d}{\left(z+i \omega_{k, \alpha}\right) \mathcal{F}(z)} \\
& -\sum_{\alpha^{\prime}=L, R} \sum_{k^{\prime}=0}^{k_{\max }} \frac{t_{k, \alpha} t_{k^{\prime}, \alpha^{\prime}}}{\left(z+i \omega_{k, \alpha}\right)\left(z+i \omega_{k^{\prime}, \alpha^{\prime}}\right)} \frac{c_{k^{\prime}, \alpha^{\prime}}}{\mathcal{F}(z)},
\end{aligned}
$$

where $d=\mathbf{d}(t=0), c_{k, \alpha}=\mathbf{c}_{k \alpha}(t=0)$, and

$$
\mathcal{F}(z)=z+i \epsilon+\sum_{\alpha=L, R} \sum_{k=0}^{k_{\max }} \frac{t_{k, \alpha}^{2}}{z+i \omega_{k, \alpha}} .
$$

The roots of $\mathcal{F}(z)$ are related to the energies of the Hamiltonian (6) by $\epsilon_{i}=i z_{i}$. The time evolution can be obtained by an inverse Laplace transformation

$$
\mathbf{o}(t)=\frac{1}{2 \pi i} \int_{\delta-i \infty}^{\delta+i \infty} e^{z t} \hat{\mathbf{o}}(z) d z
$$

where $\delta>0$ has to be chosen so that the integration contour is completely within the region of convergence of $\hat{\mathbf{o}}(z)$. In order to perform analytical calculations, we consider the system in the continuum limit (CL). For this reason, we transform the main part of the sum in $\mathcal{F}(z)$ into an integral

$$
\sum_{k \neq 0} \frac{t_{k, \alpha}^{2}}{z+i \omega_{k, \alpha}} \rightarrow \frac{1}{2 \pi} \int_{0}^{\infty} d \omega^{\prime} \frac{\Gamma_{\alpha}\left(\omega^{\prime}\right)}{z+i \omega^{\prime}} \equiv C_{\alpha}(z)
$$

with the tunnel rate $\Gamma_{\alpha}(\omega)=2 \pi \sum_{k} t_{k \alpha}^{2} \delta\left(\omega-\omega_{k \alpha}\right)$, which we assume to be analytic for $\omega>0$ in the CL. We emphasize that in order to investigate the time evolution of the condensate, it is necessary to extract the groundstate energy modes $k=0$ from the integral. In the CL, we thus have

$$
\mathcal{F}(z)=z+i \epsilon+\sum_{\alpha=L, R} \frac{t_{0, \alpha}^{2}}{z+i \omega_{0, \alpha}}+C_{\alpha}(z) .
$$

This approach is a modification of former investigations of the Fano-Anderson model as in Ref. [32. The extraction of the ground-state modes allows for a detailed analysis of the condensate dynamics and creates a three-mode system with modes $\mathbf{c}_{0, L}, \mathbf{c}_{0, R}$, and $\mathbf{d}$ which is coupled to the reservoirs. The latter are described by the tunnel rates $\Gamma_{\alpha}(\omega)$. A sketch of the resulting setup is depicted in Fig. 1(d). In Sec. IVC, we derive an effective nonhermitian Hamiltonian for the three-mode system which exactly resembles the dynamics in the long-time limit. The non-hermitian property reflects the fact that the coherent dynamics in this system is subjected to loss and is thus not unitary. Our approach thus provides the possibility to analytically study these effects. 
a) Josephson current $I_{R, J} / \omega_{c}$

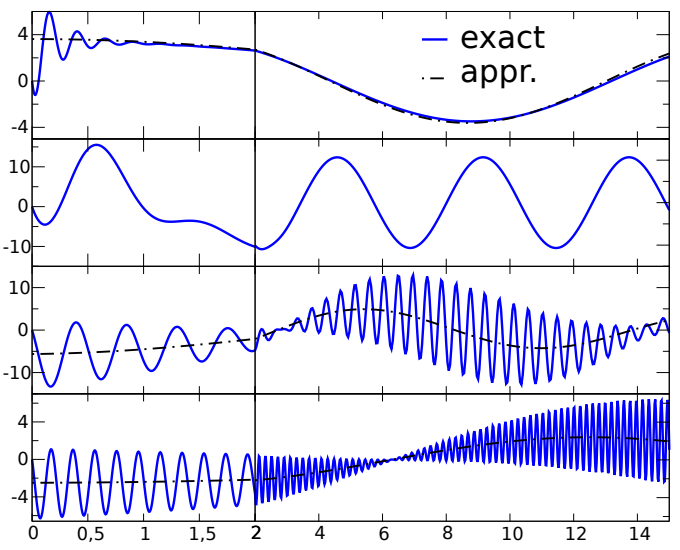

c)

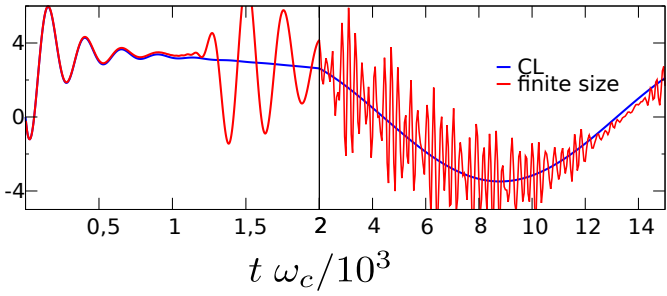

\section{b) Occupation of right reservoir}

$$
\begin{aligned}
\frac{\epsilon}{\omega_{c}} & =0.04 \\
\frac{\epsilon}{\omega_{c}} & =0.02 \\
& \gtrsim \epsilon_{c r i t} \\
\frac{\epsilon}{\omega_{c}} & =0
\end{aligned}
$$

$\frac{\epsilon}{\omega_{c}}=-0.02$

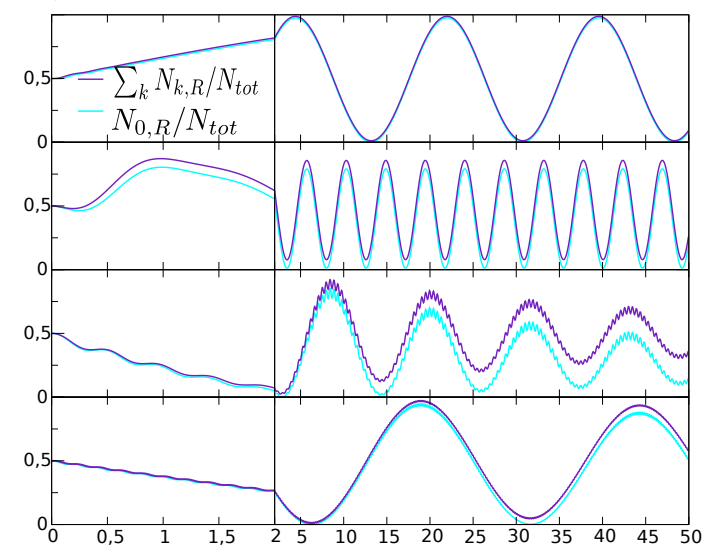

d)

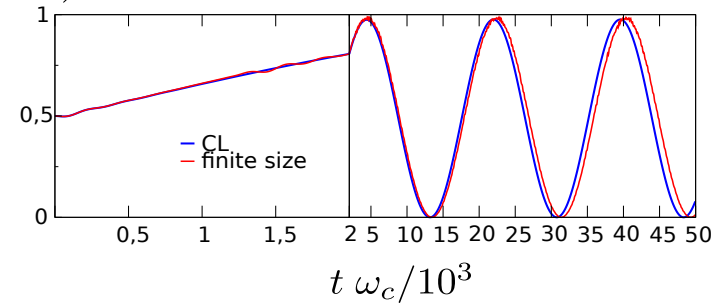

FIG. 2. (Color online) (a) Josephson current as a function of time. The parameters are the same as in Fig. 1)(c). The current shows a similar behavior as the dot occupation $N_{d}(t)$. The dash-dotted line depicts an approximation of the time-averaged current given in Eq. (49). For $\epsilon \approx \epsilon_{\text {crit }}$ the approximation completely fails (not shown). The oscillation frequencies are given by the imaginary parts of the roots of $\tilde{\mathcal{F}}(z)$ which are depicted in Fig. 3(e) and are approximately given by Eq. (35). (b) Total occupation $\sum_{k=0}^{k_{\max }} N_{k, R}$ and the ground-state occupation $N_{0, R}$ of the right reservoir. Both observables are scaled by the total particle number in the system $N_{\text {tot }}=n_{L}+n_{R}$. The fast oscillations of the current are only visible in the reservoir occupation close to the transition at $\epsilon \lesssim \epsilon_{\text {crit }}$. (c) Finite-size simulation with $k_{\max }=100$. For $t \omega_{c} \lesssim 1 \cdot 10^{3}$ the finite-size time evolution exactly agrees with the CL. The fluctuations for $t \gtrsim \omega_{c} \cdot 10^{3}$ are due to the finite energy gaps between the reservoir modes near the ground state. (d) The fluctuation are hardly visible in the ground-state occupation $N_{0, R}(t)$.

We consider a tunnel rate with an exponential cut-off in its energy dependence, thus,

$$
\Gamma_{\alpha}(\omega)=\gamma_{\alpha}\left(\frac{\omega}{\omega_{c}}\right)^{\eta} e^{-\frac{\omega}{\omega_{c}}} \Theta(\omega)
$$

where $\eta>-1$ is a scaling exponent describing the tunnel rate close to $\omega=0$. The parameter $\gamma_{\alpha}$ is the coupling constant and $\omega_{c}$ denotes the cut-off frequency. The function $\Theta(\omega)$ denotes the Heavyside function and guarantees that the reservoir spectrum is bounded at $\omega=0$. We choose this tunnel rate, as it allows for analytical calculations. However, many of our results as, e.g., the complex frequencies in Eq. (35) are expressed in terms of $\Gamma_{\alpha}(\omega)$ itself and thus hold for more general parametrizations than Eq. 211. In our investigations, we find that the dynamics of the condensate is mainly determined by the tunnel rates near $\omega \gtrsim 0$. So the exact details as the cut-off of the tunnel rate are not important for our qualitative results. For the tunnel rate (21) the integration in (19) can be performed exactly and we obtain

$$
C_{\alpha}(z)=-i \frac{\gamma_{\alpha}}{2 \pi}\left(-i \frac{z}{\omega_{c}}\right)^{\eta} e^{-i \frac{z}{\omega_{c}}} \tilde{\Gamma}(1+\eta) \tilde{\Gamma}\left(-\eta,-i \frac{z}{\omega_{c}}\right)
$$

where $\tilde{\Gamma}(x)$ and $\tilde{\Gamma}(x, z)$ denote the complete and incomplete Gamma functions, respectively. The incomplete Gamma function $\tilde{\Gamma}\left(-\eta,-i \frac{z}{\omega_{c}}\right)$ is characterized by a branch-cut discontinuity in the complex plane running from $z=-i \infty$ to $z=0$. Also the prefactor $\left(-i z / \omega_{c}\right)^{\eta}$ contributes for non-integer $\eta$ to the branch cut.

The branch cut is not a specific property of the chosen parametrization in (21), but is a generic property of $C_{\alpha}(z)$ as the integration in (19) runs over positive frequencies only. It occurs since the integrand in $(19)$ has a pole at $z=-i \omega$. In Sec. IVA we discuss how to handle these branch-cut discontinuities analytically. 


\section{Dynamical regimes}

We calculate the time evolution of the system operators by performing an exact inverse Laplace transformation of (15) and (16). The expectation values of the observables we are interested in, such as the current $I_{R, J}(t)=\operatorname{tr}\left[\mathbf{I}_{R, J}(t) \rho\right]$, depend on correlation functions, e.g., $\left\langle\mathbf{c}_{k, \alpha}^{\dagger} \mathbf{c}_{k^{\prime}, \alpha^{\prime}}\right\rangle_{0}$ at $t=0$. The expectation value $\langle\mathbf{O}\rangle_{t}$ is defined by

$$
\langle\mathbf{O}\rangle_{t} \equiv \operatorname{tr}[\mathbf{O}(t) \rho] .
$$

For the initial condition (9) in the zero-temperature limit, the only non-vanishing correlation functions are

$$
\begin{aligned}
& \left\langle\mathbf{c}_{k, \alpha}^{\dagger} \mathbf{c}_{k, \alpha}\right\rangle_{0}=\delta_{k, 0} n_{\alpha}, \\
& \left\langle\mathbf{c}_{0, R}^{\dagger} \mathbf{c}_{0, L}\right\rangle_{0}=\sqrt{n_{L} n_{R}} e^{i \Delta \phi},
\end{aligned}
$$

where $\Delta \phi=\phi_{R}-\phi_{L}$ denotes the initial phase difference of the left and right condensate, and $n_{\alpha}$ their initial occupation.

The results are depicted in Fig. 1(c) and Fig. 2. In the numerical calculations throughout the article we assume a symmetric system, meaning that $\omega_{0, L}=\omega_{0, R}=0$. In the time evolution we observe two dynamical regimes. They can be distinguished best by considering the dot occupation $N_{d}$, the Josephson current $I_{R, J}$, and the occupation of the right ground-state mode $N_{0, R}$.

In regime I for $\epsilon>\epsilon_{\text {crit }} \approx 0.016 \omega_{c}$, we observe that $N_{d}(t)$ and $I_{R, J}(t)$ exhibit initial oscillations that are quickly damped. The determination of $\epsilon_{\text {crit }}$ is discussed in Sec. IV B. For longer times, we find that $N_{d}(t)$ reaches a constant value while $I_{R, J}(t)$ performs oscillations with a very long period, which are undamped. The oscillation frequency increases when approaching $\epsilon=\epsilon_{\text {crit }}$. During these oscillations the main fraction of the particles oscillates between the two reservoir ground states. A rather small amount of the initially condensed particles are subjected to depletion. They are scattered to the excited modes during the dynamics (difference between the curves in Fig. 2(b)). The depletion is stronger for $\epsilon$ close to $\epsilon_{\text {crit }}$.

In regime II for $\epsilon<\epsilon_{\text {crit }}$, the dot occupation $N_{d}(t)$ exhibits fast oscillations which are only weakly damped. The damping is stronger close to the transition. The time evolution of $I_{R, J}(t)$ displays a superposition of two oscillations with long and short period, respectively. However, only the slow oscillations significantly change the occupation of the right reservoir in panel (b). The depletion is only noticeable close to $\epsilon_{\text {crit }}$.

In Fig. 2(c), we depict a finite-size simulation of the Josephson current as a benchmark for our approach. In appendix A. we provide information about its calculation. Thereby, each reservoir consists of a rather small number of modes, namely $k_{\max }=100$.

As we observe in Fig. 2(c), the numerical finite-size simulation agrees well with the CL calculations for short times $t \omega_{c} \cdot 10^{3} \lesssim 1$ after which the finite-size simulation starts to exhibit deviations. These appear due to the finite energy spacing $\delta \omega \approx \omega_{c} / k_{\max }$ between the levels close to the ground state. In numerical studies we find that the starting time of these deviations $T_{d e v}$ grows for increasing $k_{\max }$.

However, we assume that these fluctuations are not particularly relevant in experiments. The actual observable quantity is the particle number in the right reservoir which is mainly given by the condensate particles $N_{0, R}$. As we see in Fig. 2(d), the fluctuations in the current are averaged so that they are hardly visible in $N_{0, R}$. Additionally, weak interactions which are always present in experiments could induce a damping of these finite-size fluctuations.

Finally, we emphasize that the two regimes I and II are connected by a smooth crossover as a function of $\epsilon$ for finite $t_{0, \alpha}$. This becomes clear when considering the relation of observables and roots of the system later in this article in Sec. IVD, The transition is only nonanalytic in the limit $t_{0, \alpha} \rightarrow 0$.

\section{LONG-TIME LIMIT}

\section{A. Analysis in the complex plain}

In order to better understand the time evolution in Fig. 2, we investigate the time evolution for intermediate and long times in more detail. To this end, we have to identify the main contributions in the inverse Laplace transformation of (15) and (16). In particular, the analytic properties of $\overline{\mathcal{F}}(z)$ and its roots are important for the inverse Laplace transform so that we analyze it in the following.

First, we consider the roots of $\mathcal{F}(z)$ in $(17)$ for a finitesized system with $k_{\max }$ states in the reservoirs. The roots of $\mathcal{F}(z)$ are located on the imaginary axis as sketched in Fig. 3(a). Using the residue theorem with the dashed contour shown in Fig. 3(a), we find that one can perform the inverse Laplace transformation $(18)$ for $\mathbf{o}=\mathbf{d}, \mathbf{c}_{k \alpha}$ corresponding to the blue integration contour, by evaluating the residua

$$
\mathbf{o}(t)=\sum_{a \in D_{f s}} \operatorname{Res}_{z=a} \quad e^{z t} \hat{\mathbf{o}}(z),
$$

where $D_{f s}$ is the set of all poles in (15) and $(16)$ which includes the set of all roots of $\mathcal{F}(z)$.

For $k_{\max } \rightarrow \infty$, the roots move closer to each other and finally form the branch cut of $\mathcal{F}(z)$ in 20 . The branch cut is depicted in Fig. 3(b). Everywhere else in the complex plane, $\mathcal{F}(z)$ is analytic. The branch cut of $\mathcal{F}(z)$ is due to the branch cut of $C_{\alpha}(z)$, c.f. Eq. (22). At the branch cut, the function $C_{\alpha}(z)$ has a jump discontinuity of

$$
\lim _{\delta \downarrow 0} C_{\alpha}(-i \omega+\delta)-C_{\alpha}(-i \omega-\delta)=\Gamma_{\alpha}(\omega)
$$



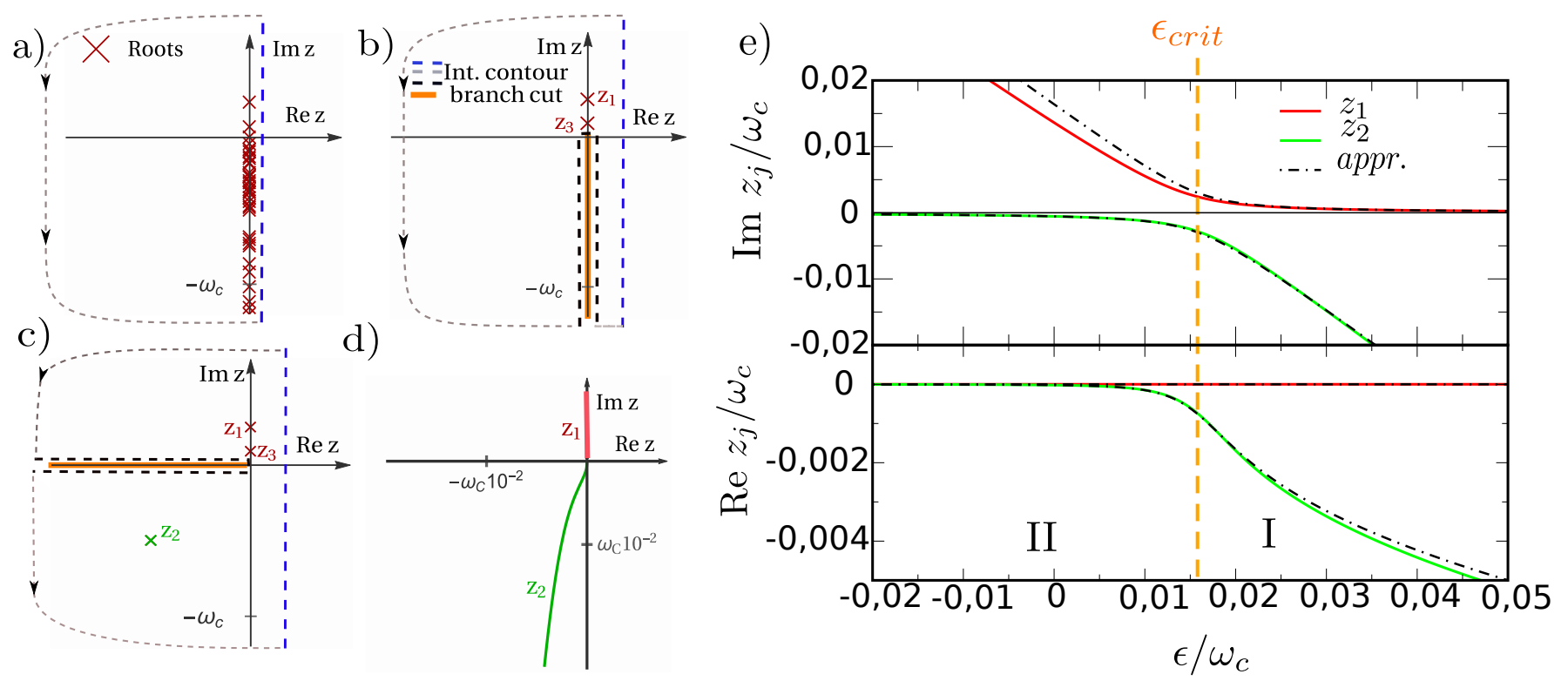

FIG. 3. (Color online) (a) Sketch of the roots of $\mathcal{F}(z)$ in Eq. (17) for a finite-system with $k_{\text {Max }}=100$. The dashed curve depicts the contour used to apply the residue theorem. See main text for more details. (b) In the CL, the roots get dense and finally form a branch cut (orange) located at the negative imaginary axes. In addition, there are two roots $z_{1}$ and $z_{3}$ generated by the extraction of the ground-state modes in Eq. 20). The black dashed curve depicts the integration contour $\mathcal{C}$ needed to evaluate Eq. 28). (c) Rotation of the branch cut so that its contribution in the inverse Laplace transformation 28 is negligible in the long-time limit. Due to the rotation, the modified $\tilde{\mathcal{F}}(z)$ given in 30 exhibits an additional root with finite real part $z_{2}$. (d) Trajectories of $z_{1}$ and $z_{2}$ in the complex plain as function of $\epsilon$. The parameters are identical to Fig. 1(c) so that $z_{3} \rightarrow 0$. (e) Real and imaginary part of the roots $z_{1}$ and $z_{2}$ as a function of $\epsilon$. The dashed line depicts the approximation 35 .

where $\Gamma_{\alpha}(\omega)$ is the tunnel rate and $\omega>0, \delta \in \mathbb{R}$. This relation can be proven using Eq. (19) and the Dirac identity

$$
\lim _{\delta \downarrow 0} \frac{1}{\left(\omega-\omega^{\prime}\right) \pm i \delta}=P \frac{1}{\omega-\omega^{\prime}} \mp i \pi \delta\left(\omega-\omega^{\prime}\right),
$$

where $P$ denotes the principal value.

Besides, for the chosen parameters in Fig. 3(b) there are two roots $z_{1}$ and $z_{3}$ which are not merged with the branch cut. They appear due to the extraction of the ground states as explained in Eq. 200.

As for the finite-size system, we apply the residue theorem with the dashed contour shown in Fig. 3(b) in order to evaluate the inverse Laplace transformation (18). The integration contour is chosen so that the surrounded area is analytic except of isolated poles. In doing so, we find

$$
\mathbf{o}(t)=\sum_{a \in D} \operatorname{Res}_{z=a} \quad e^{z t} \hat{\mathbf{o}}(z)-\frac{1}{2 \pi i} \int_{\mathcal{C}} e^{z t} \mathbf{o}(z) d z
$$

where $\mathcal{D}$ is the set of all isolated poles appearing in 15 and 16 which includes the roots of $\mathcal{F}(z)$ in 200 . Formula (28) is valid under the assumption of a vanishing integrand for $\operatorname{Re} z \rightarrow-\infty$. For this reason, we can omit the gray integration contour in Fig. 3(b). The only remaining integration contour is $\mathcal{C}$ depicted in black in Fig. $3(\mathrm{~b})$ and encircles the branch cut of $\mathcal{F}(z)$. Equation (28) constitutes an exact expression in the CL. However, the evaluation of the branch-cut integral in Eq. (28) is numerically expensive and analytically unfavorable. For this reason, we explain in the following how to circumvent this problem in the long-time limit.

The branch cut of $C_{\alpha}(z)$ in 19 which generates the branch cut of $\mathcal{F}(z)$ is not uniquely defined. There is the possibility to modify $C_{\alpha}(z)$ so that its branch cut is located elsewhere. As the branch cut separates the bottomleft and the bottom-right sector of the complex plane, we modify $C_{\alpha}(z)$ in the bottom-left sector, so that the current branch cut is displaced and the modified function $\tilde{C}_{\alpha}(z)$ is analytic on the negative imaginary axis. More, precisely, that modification reads

$$
\tilde{C}_{\alpha}(z)=C_{\alpha}(z)+ \begin{cases}\Gamma_{\alpha}(i z) & \operatorname{Re} z<0 \wedge \operatorname{Im} z<0 \\ 0 & \text { else }\end{cases}
$$

where $\Gamma_{\alpha}(i z)$ is the analytic continuation of the tunnel rate as defined for $\omega=i z>0$ onto the complex plane. One can find from Eq. (26) that $\tilde{C}_{\alpha}(z)$ is continuous along the previous branch cut position. In appendix B we prove, that $\tilde{C}_{\alpha}(z)$ is indeed analytic on the negative imaginary axis. In addition, as $\tilde{C}_{\alpha}(z)$ is a sum of analytic functions in the bottom-left sector, it is analytic there. Yet, due to the modification, $\tilde{C}_{\alpha}(z)$ is not continuous on the negative real axis separating the top-left and bottomleft sector as depicted in Fig. 3(c). Consequently, $\tilde{C}_{\alpha}(z)$ has now a branch cut there. Thus, $C_{\alpha}(z)$ and $\tilde{C}_{\alpha}(z)$ are related by a branch cut rotation. 
Consequently, we also modify

$$
\tilde{\mathcal{F}}(z) \equiv \mathcal{F}(z)-\sum_{\alpha=L, R} C_{\alpha}(z)+\sum_{\alpha=L, R} \tilde{C}_{\alpha}(z),
$$

which is therefore also analytic everywhere except on the negative real axis. As $\tilde{\mathcal{F}}(z)=\mathcal{F}(z)$ for $\operatorname{Re} z>0$, the inverse Laplace transformation in Eq. (18) is not affected so that the operators as a function of time remain invariant under the branch-cut rotation. As before, we employ the residue theorem to simplify the evaluation of the inverse Laplace transformation in Eq. (18) with the blue integration contour in Fig. 3(c). The corresponding contour is depicted by the dashed lines in Fig. 3(c). The result is formally equivalent to Eq. 28), but with the integration contour $\mathcal{C}$ depicted in black in Fig. 3(c).

$\tilde{\mathcal{F}}(z)$ is different from $\mathcal{F}(z)$ in the third sector of the complex plain. This gives rise to an additional root $z_{2}$ with negative real part. We depict it also in Fig. 3(c). In the limit of $t_{k, \alpha} \rightarrow 0$, it corresponds to the gate potential $\epsilon=i z_{2}$ in regime I. For the symmetric system, we calculate the leading orders of the position of the root for small $t_{\alpha, 0}$ in appendix $\mathrm{D}$. Altogether, $\tilde{\mathcal{F}}(z)$ possesses three roots. We found that the number of roots of $\tilde{\mathcal{F}}(z)$ is independent of the system parameters.

We approximate now the inverse Laplace transformation by neglecting the branch-cut integral in Eq. 287. This is justified as the integrand in (28) contains the factor $e^{z t}$, which vanishes in the long-time limit as the integration contour surrounds the negative real axis. For example, the branch-cut integral $\mathcal{I}_{b c}$ belonging to the second line in Eq. (16) for $k, k^{\prime}=0$ is for long times approximately given by

$$
\mathcal{I}_{b c} \approx \frac{1}{2 \pi i} \frac{\sum_{\alpha} \gamma_{\alpha}}{4 t_{0}^{2}}\left(\frac{-i}{\omega_{c}}\right)^{\eta} \tilde{\Gamma}(\eta+1) \frac{1}{t^{\eta+1}} c_{0, \alpha^{\prime}},
$$

if $\omega_{0, \alpha}=\omega_{0, \alpha^{\prime}}=0$ and $t_{0, L}=t_{0, R}=t_{0}$ which is the most important case in our article. In appendix $\mathrm{C}$, we provide more information about the calculation. There, we also consider the cases $\omega_{0, \alpha} \neq \omega_{0, \alpha^{\prime}}=0$ and $\omega_{0, \alpha} \neq 0 \neq \omega_{0, \alpha^{\prime}}$ which yield similar results. From (31) we see, that the branch cut integral vanishes algebraically in time for long times if $\eta>-1$. Branch-cut integrals corresponding to the other terms in (15) and (16) vanish even faster.

For intermediate times we found that the branch-cut integral contributes only insignificantly for $\epsilon$ away from the transition point $\epsilon_{\text {crit }}$. As a result, the dynamics of the system for intermediate and long times is determined by the poles of $(15)$ and $(16)$, which we can efficiently calculate numerically.

Finally, we have to point out a subtlety. The function $\tilde{\mathcal{F}}(z)$ exhibits only three roots for $\omega_{0, L} \neq \omega_{0, R}$. For the special case $\omega_{0, L}=\omega_{0, R}$ which we mainly focus on in this article, $\tilde{\mathcal{F}}(z)$ has indeed only two roots. The missing root corresponds to a dark state with energy $\omega_{0, L}$. The corresponding mode reads

$$
\mathbf{c}_{\text {dark }}=\frac{1}{\sqrt{t_{0, L}^{2}+t_{0, R}^{2}}}\left(t_{0, L} \mathbf{c}_{0, R}-t_{0, R} \mathbf{c}_{0, L}\right) .
$$

This mode obviously does not couple to the dot or the excited reservoir modes. For this reason, it does not appear in $\tilde{\mathcal{F}}(z)$. It is not hard to show that $\left[\mathbf{H}, \mathbf{c}_{\text {dark }}^{\dagger} \mathbf{c}_{\text {dark }}\right]=0$. Therefore, the particle number in the dark mode remains constant and it is not subjected to particle loss. Consequently, there is no complete depletion of the groundstate modes if the dark state is initially occupied. In our generic investigation in Sec. IVC we consider the more general case of $\omega_{0, L} \neq \omega_{0, R}$ and regard the equality as the limit $\omega_{0, L} \rightarrow \omega_{0, R}$.

Furthermore, if additionally $\omega_{0, L} \rightarrow \omega_{0, R}=0$, than the dark-state root $z_{3} \rightarrow 0$, which lies within the branchcut contour $\mathcal{C}$. For this reason, we treat this special case formally with a limit procedure: first we assume $\omega_{0, L} \rightarrow \omega_{0, R} \neq 0$ and than take the limit $\omega_{0, R} \rightarrow 0$ after performing the inverse Laplace transformation.

\section{B. Roots of the symmetric system}

In the following, we analyze the roots of $\tilde{\mathcal{F}}(z)$ for the symmetric system with $\omega_{0, L}=\omega_{0, R}=0$. For simplicity we also assume symmetric tunneling rates $t_{0, L}=t_{0, R} \equiv$ $t_{0}$. Here, we give an analytical expression for the leading contributions of the real and imaginary part of the roots $z_{1}$ and $z_{2}$.

In order to express the location of the roots, we define the real and the imaginary part of $\sum_{\alpha} \tilde{C}_{\alpha}(z)$ by

$$
\lim _{\delta \downarrow 0} \sum_{\alpha=L, R} \tilde{C}_{\alpha}(-i \omega+\delta) \equiv \Gamma(\omega)+i \Sigma(\omega),
$$

with $\omega, \delta \in \mathbb{R}$. The real part can be expressed with the tunnel rates

$$
\Gamma(\omega)=\frac{1}{2}\left(\Gamma_{L}(\omega)+\Gamma_{R}(\omega)\right) .
$$

which can be proven using the Dirac identity (27).

In appendix D we show that for small $t_{0}$ and $\eta>0$ the roots $z_{1}$ and $z_{2}$ of $\tilde{\mathcal{F}}(z)$ are approximately located at

$$
z_{j} \approx z_{j}^{0}-\frac{z_{j}^{0} \Gamma\left(i z_{j}^{0}\right)}{2 z_{j}^{0}+i[\epsilon+\Sigma(0)]},
$$

where the imaginary part $z_{j}^{0}$ reads

$$
z_{1,2}^{0}=-i \frac{1}{2}\left(\epsilon+\Sigma(0) \pm \sqrt{(\epsilon+\Sigma(0))^{2}+8 t_{0}^{2}}\right) .
$$

Using Eq. 22 we find for the Lamb shift

$$
\Sigma(0)=-\sum_{\alpha=L, R} \frac{\gamma_{\alpha}}{2 \pi} \tilde{\Gamma}(\eta),
$$

which renormalizes the gate potential $\epsilon$. The second term in Eq. (35) is the leading order of the real part. Interestingly, it is proportional to $\Gamma\left(i z_{j}^{0}\right)$. Consequently, if $i z_{j}^{0}<0$, the real part vanishes due to Eq. 21). 
The analytical expressions for $z_{1}$ and $z_{2}$ are depicted in Fig. 3(e) and agree well with the numerical calculation. The imaginary parts of the roots $z_{j}$ can be used to define the transition between the two dynamical regimes I and II. For $t_{0} \rightarrow 0$, the two roots get degenerate for

$$
\epsilon=\epsilon_{\text {crit }} \equiv-\Sigma(0) .
$$

This relation defines the regime I for $\epsilon>\epsilon_{\text {crit }}$ and regime II for $\epsilon<\epsilon_{\text {crit }}$. As we see in Fig. 3(e), the root $z_{2}$ has the property $\operatorname{Re} z_{2}<0$. In the regime II we have $\operatorname{Re} z_{2} \approx 0$ which vanishes exactly for $t_{0}=0$ as we can see from (35). Yet, in regime I it is always finite. By contrast, the real part of $z_{1}$ is always $\operatorname{Re} z_{1}=0$. To clarify the dependence of $z_{1}$ and $z_{2}$ on $\epsilon$, we also depict the trajectory of these roots in the complex plain as a function of $\epsilon$ in Fig. 3 (d).

\section{Effective Hamiltonian}

We are interested in the dynamics of observables which can be expressed by the operators $\mathbf{d}, \mathbf{c}_{0, L}$, and $\mathbf{c}_{0, R}$, such as the dot occupation or the Josephson current. As we have assumed a zero-temperature limit at time $t=0$, the only relevant operators in Eqs. (15) and (16) at $t=0$ are even these operators. For this reason, we can effectively restrict the BEC dynamics to a three-mode system.

For a notational reason we define

$$
\mathbf{v}_{t} \equiv\left(\begin{array}{c}
\mathbf{d}(t) \\
\mathbf{c}_{0, L}(t) \\
\mathbf{c}_{0, R}(t)
\end{array}\right)
$$

The dynamics of $\mathbf{v}_{t}$ in the long-time limit is determined by an effective non-hermitian Hamiltonian, which fulfills

$$
i \frac{d}{d t} \mathbf{v}_{t}=\mathcal{H}_{e f f} \mathbf{v}_{t}
$$

which is formally equivalent to a single-particle Schrödinger equation. Thereby, the effective Hamiltonian $\mathcal{H}_{\text {eff }}$ reads

$$
\mathcal{H}_{\text {eff }}=S D S^{-1}
$$

where $D=\operatorname{diag}\left[i z_{1}, i z_{2}, i z_{3}\right]$ is a diagonal matrix containing the roots of $\tilde{\mathcal{F}}(z)$. The columns of the matrix $S$ are given by $S_{j}=w_{j}$ with

$$
w_{j}=\frac{1}{\sqrt{\zeta_{j}}}\left(-1, \frac{t_{0, L}}{\left(\omega_{0, L}-i z_{j}\right)}, \frac{t_{0, R}}{\left(\omega_{0, R}-i z_{j}\right)}\right)^{T},
$$

where $\zeta_{j}$ accounts for the normalization. We note that the $w_{j}$ are in general not orthogonal to each other. Additionally, the roots $z_{j}$ can be complex-valued so that the effective Hamiltonian is non-hermitian.

We remark that the only important poles $z_{i}$ for the effective Hamiltonian are the ones given by $\tilde{\mathcal{F}}(z)=0$. The other poles appearing in (15) and (16) such as $z=-i \omega_{0, \alpha}$ are not relevant. More precisely, the factors $\left(z+i \omega_{0, \alpha}\right)$ appearing in the nominators can be combined with $\tilde{\mathcal{F}}(z)$, which cancels the nominators in the third term of 20 . This combination therefore reveals that the first order pole at $z=-i \omega_{0, \alpha}$ is not an actual pole in second term of (15) and in the second and third term of 16). Finally, the pole of the first term in (16) at $z=-i \omega_{0, \alpha}$ is annihilated by the term with $\left(z+i \omega_{0, \alpha}\right)^{2}$ in the nominator of the third term in (16) during the inverse Laplace transformation.

We note that effective non-hermitian Hamiltonians can give rise to interesting effects not present in hermitian systems. A particular appealing effect is a non-hermitian degeneracy, at which two eigenvalues and their corresponding eigenstates merge which is denoted as an exceptional point $[22,25]$. Usually, the construction of non-hermitian Hamiltonians includes phenomenological assumptions. Here, we presented a completely microscopic derivation which can be used to study the fate of exceptional points under more realistic conditions. In particular, here the eigenvalues are not determined by the roots of the characteristic polynomial of the Hamiltonian, but by the roots of $\tilde{\mathcal{F}}(z)=0$, which exhibits a non-linearity due to $\tilde{C}_{\alpha}(z)$. This might gives rise to a qualitatively different behavior of the system at or close to the exceptional points.

In the remainder of this section we prove that $\mathcal{H}_{\text {eff }}$ generates indeed the correct dynamics in the long-time limit. After neglecting the branch cut in Eq. (28), the time evolution can be evaluated by calculating the corresponding residua at the roots of $\tilde{\mathcal{F}}(z)$. In doing so, the time evolution of the operators reads

$$
\mathbf{v}_{t}=\sum_{z_{j} \in \mathcal{D}_{\mathcal{F}}} e^{z_{j} t} Q\left(z_{j}\right) \mathbf{v}_{0}
$$

where we define $\mathcal{D}_{\mathcal{F}}=\{z \mid \tilde{\mathcal{F}}(z)=0\}$ which is the set of all three roots of $\tilde{\mathcal{F}}(z)=0$. Here $Q\left(z_{j}\right)$ denotes a $3 \times 3$ matrix and reads

$$
\begin{aligned}
& Q\left(z_{j}\right)=R_{z_{j}} \times \\
& \left(\begin{array}{ccc}
1 & \frac{-t_{0, L}}{\left(\omega_{0, L}-i z_{j}\right)} & \frac{-t_{0, R}}{\left(\omega_{0, R}-i z_{j}\right)} \\
\frac{-t_{0, L}}{\left(\omega_{0, L}-i z_{j}\right)} & \frac{t_{0, L}^{2}}{\left(\omega_{0, L}-i z_{j}\right)^{2}} & \frac{t_{0, R} t_{0, L}}{\left(\omega_{0, R}-i z_{j}\right)\left(\omega_{0, L}-i z_{j}\right)} \\
\frac{-t_{0, R}}{\left(\omega_{0, R}-i z_{j}\right)} & \frac{t_{0, R} t_{0, L}}{\left(\omega_{0, R}-i z_{j}\right)\left(\omega_{0, L}-i z_{j}\right)} & \frac{t_{0, R}^{2}}{\left(\omega_{0, R}-i z_{j}\right)^{2}}
\end{array}\right)
\end{aligned}
$$

with

$$
R_{z_{j}}=\operatorname{Res}_{z=z_{j}} \frac{1}{\tilde{\mathcal{F}}(z)} .
$$

The matrix $Q\left(z_{j}\right)$ is hermitian for imaginary $z_{j}$. Interestingly, it fulfills a projector-like relation

$$
Q\left(z_{j}\right) Q\left(z_{j}\right)=Q\left(z_{j}\right) \zeta_{z_{j}} R_{z_{j}} .
$$

This relation even holds for complex-valued $z_{j}$. Consequently, two eigenvalues of $Q\left(z_{j}\right)$ are zero. The nonvanishing eigenvalue is $\zeta_{z_{j}} R_{z_{j}}$. For $\gamma_{\alpha}=0$ we have 
a bare three-mode system without coupling to the excited modes. For this reason, the eigenvalue is necessarily $\zeta_{z_{j}} R_{z_{j}}=1$, so that the time evolution is unitary. Due to the coupling to the excited reservoir modes for $\gamma_{\alpha}>0$, it is possible that $\zeta_{z_{j}} R_{z_{j}} \neq 1$. This eigenvalue thus includes information about the transient dynamics.

The normalized eigenstates corresponding to $R_{z_{j}} \zeta_{z_{j}}$ are the $w_{j}$ given in Eq. 42. Consequently, the matrix $Q\left(z_{j}\right)$ can be written as

$$
Q\left(z_{j}\right)=\zeta_{z_{j}} R_{z_{j}} w_{j} w_{j}^{T} .
$$

Inserting this into Eq. (43) and using that $w_{j}$ is an eigenstate of $\mathcal{H}_{\text {eff }}$, we can verify that Eq. 43 fulfills the Schrödinger equation 40 .

\section{Relation of observables and roots}

The properties of the roots are reflected in the oscillations of the observables. For example, the Josephson current for long times reads

$$
I_{R, J}(t)=2 \operatorname{Re} \sum_{j, j^{\prime}} e^{\left(z_{j}^{*}+z_{j^{\prime}}\right) t} \tilde{I}_{R, J}^{C}\left(z_{j}, z_{j^{\prime}}\right)
$$

where the constants $I_{R, J}^{C}\left(z_{j}, z_{j^{\prime}}\right)$ are a function of the roots and depend on the initial condition. Their explicit expressions can be found in Eq. E2). The time evolution of other observables such as the ground-state occupation of the right reservoir $N_{0, R}(t)$ read similarly. We see that the oscillations are determined by the exponential factor $e^{\left(z_{j}^{*}+z_{j^{\prime}}\right) t}$. We find that $I_{R, J}^{C}\left(z_{j}, z_{j}\right)=0$. For the symmetric system $\omega_{0, \alpha}=0$ and $t_{0, \alpha}=t_{0}, I_{R, J}^{C}\left(z_{j}, z_{j^{\prime}}\right)$ is rather small if both $z_{j}, z_{j^{\prime}} \neq z_{3}=0$. So the most important terms are the ones where one root is $z_{1}$ or $z_{2}$ and the other is $z_{3}=0$. Thus, the dynamics is mainly determined by the roots $z_{1}$ and $z_{2}$. Thereby, the imaginary parts are responsible for the oscillation frequencies while the real parts determine the damping. The oscillatory behavior of other observables such as the ground-state occupation of the right reservoir $N_{0, R}(t)$ or the dot occupation $N_{d}(t)$ is similarly determined by the roots $z_{1}$ and $z_{2}$.

As a consequence of the finite real part of $z_{2}$ in regime I, the fast oscillations in the observables caused by the imaginary part of $z_{2}$ are strongly damped as can be seen in Fig.2(a) for $\epsilon=0.04 \omega_{c}$. The oscillations of $I_{R, J}$ with a long period are caused by $z_{1}$, as it has a small imaginary part. They are undamped as $\operatorname{Re} z_{1}=0$.

In regime II, the imaginary part of $z_{1}$ is large. For this reason we observe fast oscillations which are undamped. The imaginary part of $z_{2}$ is small so that it causes oscillations with a long period. However, there is a very small damping due to the very small real part of $z_{2}$. This can be seen best in $N_{0, R}(t)$ in Fig. 2(b) for $\epsilon=0$.

Due to the damping describing the particle loss in the condensate, the excited reservoir modes get occupied. As $\operatorname{Re} z_{2}$ is quite large in regime $\mathrm{I}$, this condensate depletion

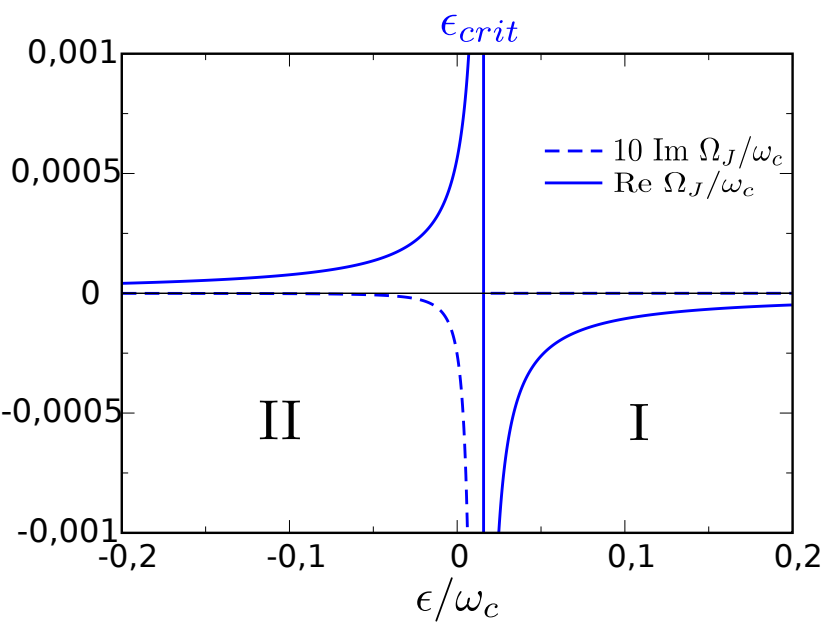

FIG. 4. (Color online) Characteristic frequency $\Omega_{J}$ appearing in the low-frequency Josephson current Eq. (49). The parameters are the same as in Fig. 2. In the immediate vicinity of $\epsilon_{\text {crit }}$, the expression (49) is not valid as the oscillation frequencies of the Josephson current are of the same order.

is completed after a rather short time as can bee seen in Fig. 2(b) for $\epsilon=0.02 \omega_{c}$. By contrast, due to a small Re $z_{2}$ in regime II, the damping continues even for long times, so the fraction of the particles in the excited modes keeps growing as can be observed in Fig. 2(b) for $\epsilon=0$ and $\epsilon=-0.02 \omega_{c}$. We recall that the particles which have been initially in the dark mode Eq. (32) are not subjected to depletion.

As the roots are a smooth function of the gate potential $\epsilon$ for finite $t_{0}$, c.f.Eq. (36) and Fig 3(e), and as the time dependence of the observables is closely related to the roots, the crossover in the time evolution of the observables from regime I to regime II is also smooth. Only in the limit $t_{0} \rightarrow 0$ the crossover in the dynamics gets non-analytic which we used to define $\epsilon_{\text {crit }}$ in Sec. IVB.

\section{E. Low-frequency current}

As we can observe in Fig. 2(b), the fast oscillations in the current in panel (a) in regime II are averaged in time and induce only small variations in the particle number $N_{0, R}(t)$. For this reason, we investigate the timeaveraged dynamics in the following. Here we return to the symmetric system, meaning that $\omega_{k, L} \rightarrow \omega_{k, R}=0$ and $t_{0, L}=t_{0, R} \equiv t_{0}$.

The Josephson current for long times can be expanded in terms of its frequency contributions, c.f. Eq. (48). We define the low-frequency current by keeping only the contribution with the smallest frequency. Formally, this corresponds to a moving time averaged with a time window $\tau$ around a time $t$. The duration $\tau$ has to be chosen so that $2 \pi / \tau$ is smaller than all frequencies except one. As we see in Fig. 2(a) for $\epsilon=0$ and $\epsilon=-0.02 \omega_{c}$, this is 
possible as the oscillation frequencies differ considerably away from the transition at $\epsilon=\epsilon_{\text {crit }}$.

In the limit of small $t_{0}$, the low-frequency Josephson current approximately reads

$$
\begin{aligned}
\bar{I}_{R, J}(t) & \approx \frac{1}{2} \operatorname{Im}\left[\Omega_{J} e^{-i \Omega_{J} t}\right]\left(n_{R}-n_{L}\right) \\
& +\operatorname{Re}\left[\Omega_{J} e^{-i \Omega_{J} t}\right] \sin \Delta \phi \sqrt{n_{L} n_{R}},
\end{aligned}
$$

where

$$
\Omega_{J}= \begin{cases}i z_{1} & \epsilon>\epsilon_{\text {crit }} \\ i z_{2} & \epsilon<\epsilon_{\text {crit }}\end{cases}
$$

denotes the characteristic frequency. Thus, the lowfrequency current is determined by the root $z_{j}$ that has the smaller imaginary part, c.f. Fig. 3(e). Details of the derivation can be found in appendix $\left[\mathrm{E}\right.$. For $\epsilon \approx \epsilon_{\text {crit }}$, Eq. (49) fails as there is no clear separation of oscillation frequencies because $\operatorname{Im} z_{1}$ and $\operatorname{Im} z_{2}$ are in the same order of magnitude, c.f. Fig. 3(e). For comparison, we have included the analytic expression 49 in Fig. 2(a). We observe that it indeed resembles the time-averaged current away from $\epsilon_{\text {crit }}$.

Relation (49) is strongly reminiscent of Eq. (5). In contrast, here the characteristic frequency $\Omega_{J}$ is complex valued in regime II due to the depletion. In regime I, $\Omega_{J}$ is purely real-valued so that Eq. 49 exactly resembles Eq. (5).

As we can see in Eq. 49, , the characteristic frequency $\Omega_{j}$ determines the dynamics of the current. We observe in Fig. 4 that the imaginary part of $\Omega_{J}$, is considerably smaller than the real part. Thus, the Josephson current is essentially proportional to the latter. For this reason, by analyzing $\operatorname{Re} \Omega_{j}$ we gain quantitative information about the Josephson current. Expanding Re $\Omega_{J}$ using Eq. (36) for small $t_{0} /(\epsilon+\Sigma(0))$ we get

$$
\operatorname{Re} \Omega_{J} \approx-\frac{2 t_{0}^{2}}{\epsilon+\Sigma(0)}
$$

As we see from this relation and from Fig. 4, Re $\Omega_{J}$ depends sensitively on the gate potential $\epsilon$. By tuning it close or far from the transition $\epsilon_{\text {crit }}$ we have thus a large or small Josephson current. This thus provides the possibility to control the current via the gate potential. This property enables to use this system as a transistor. Moreover, one can additionally control the direction of the current. It depends on the sign of Re $\Omega_{J}$ and consequently on the sign of $\epsilon-\epsilon_{\text {crit }}$ as can be also seen in Fig. 4 .

It is worth to mention that a finite imaginary part of $\Omega_{J}$ induces an additional phase shift to $\bar{I}_{R, J}(t)$. For the chosen parameters in Fig. 4. this is rather small as the ratio of imaginary and real part of $\Omega_{J}$ is of the order of $10^{-2}$. Motivated by Eq. (4), we also investigate the relation between the correlation function
$\mathcal{C}_{R L}(t)=\left\langle\mathbf{c}_{0, R}^{\dagger} \mathbf{c}_{0, L}\right\rangle_{t}$ and the Josephson current. This somehow technical analysis is included into appendix $\mathrm{F}$.

We also find an approximate expression describing the main contribution of the time-averaged dot occupation $\bar{N}_{d}(t)$. We find that the main contribution for long times is given by

$$
\bar{N}_{d}(t) \approx \frac{\left|\Omega_{J}\right|^{2}}{4 t_{0}^{2}} e^{2 t \operatorname{Im} \Omega_{j}}\left[n_{R}+n_{L}+2 \cos \Delta \phi \sqrt{n_{L} n_{R}}\right] .
$$

This expression also agrees with the exact calculation depicted in Fig. 1(d) in regime I and resembles a main contribution of the moving time-average in regime II. Interestingly, in regime II the time-averaged dot occupation vanishes for $t \rightarrow \infty$ as a result of the finite real part of $z_{2}$. However, there are additional contributions to $\bar{N}_{d}(t)$ so that the dot occupation does not fully vanish.

\section{CONCLUSIONS}

The methods which we presented in this article provide an efficient and accurate tool to determine numerically and analytically the coherent dynamics of BoseEinstein condensates in the Fano-Anderson model. We showed that the Josephson current sensitively depends on the gate potential like in a transistor. In particular, we predict a crossover from a regime with a constant dot occupation to a regime with an oscillating one. This transition is also visible in the Josephson current between the reservoirs. The regimes appear as the energy of the reservoir modes is bounded at $\omega=0$. As a consequence the energy of the eigenstates generating the dynamics can be complex-valued, which causes a qualitatively different damping depending on the regime. Furthermore, we provide analytical expressions for observables.

Additionally, we demonstrated how to derive an effective non-hermitian Hamiltonian that exactly describes the time evolution in the long-time limit. Its complex eigenvalues as a function of the gate potential become nearly degenerate at a critical value $\epsilon=\epsilon_{c}$. This is analogous to the branching behavior in exceptional points and explains the transition between the two regimes of the time evolution in our system.

An important point to address in the future is how interactions between the particles influence the dynamics. The interactions could be introduced as in Refs. 34, 35], which investigates the equilibrium properties of a bosonic single-impurity Anderson model. Such kind of investigations could reveal the stability of the Josephson current in the presence of interactions.

\section{ACKNOWLEDGMENTS}

The authors gratefully acknowledge financial support from the DFG Grants No. BR 1528/8, No. BR 1528/9, No. SFB 910, No. GRK 1558 and SCHA 1646/3-1. 
[1] S. Krinner, D. Stadler, D. Husmann, J.-P. Brantut, and T. Esslinger, Nature (London) 517, 64 (2015).

[2] J.-P. Brantut, J. Meineke, D. Stadler, S. Krinner, and T. Esslinger, Science 337, 1069 (2012).

[3] J.-P. Brantut, C. Grenier, J. Meineke, D. Stadler, S. Krinner, C. Kollath, T. Esslinger, and A. Georges, Science 342, 713 (2013).

[4] S. Levy, E. Lahoud, I. Shomroni, and J. Steinhauer, Nature (London)) 449, 579 (2007).

[5] F. Jendrzejewski, S. Eckel, N. Murray, C. Lanier, M. Edwards, C. J. Lobb, and G. K. Campbell, Phys. Rev. Lett. 113, 045305 (2014)

[6] M. Albiez, R. Gati, J. Fölling, S. Hunsmann, M. Cristiani, and M. K. Oberthaler, Phys. Rev. Lett. 95, 010402 (2005)

[7] C. Ryu and M. Boshier, New J. of Phys. 17, 092002 (2015).

[8] J. A. Stickney, D. Z. Anderson, and A. A. Zozulya, Phys. Rev. A 75, 013608 (2007).

[9] R. A. Pepino, J. Cooper, D. Z. Anderson, and M. J. Holland, Phys. Rev. Lett. 103, 140405 (2009).

[10] D. J. Papoular, L. P. Pitaevskii, and S. Stringari, Phys. Rev. Lett. 113, 170601 (2014)

[11] D. Papoular, L. Pitaevskii, and S. Stringari, arXiv:1510.02618 (2015).

[12] C. Nietner, G. Schaller, and T. Brandes, Phys. Rev. A 89, 013605 (2014).

[13] G. Schaller, C. Nietner, and T. Brandes, New J. of Phys. 16, 125011 (2014).

[14] F. Gallego-Marcos, G. Platero, C. Nietner, G. Schaller, and T. Brandes, Phys. Rev. A 90, 033614 (2014).

[15] G. Kordas, D. Witthaut, and S. Wimberger, Ann. Phys. (Leipzig) 527, 619 (2015).

[16] D. Haag, D. Dast, H. Cartarius, and G. Wunner, Phys. Rev. A 92, 053627 (2015).

[17] B. D. Josephson, Phys. Lett. 1, 251 (1962).

[18] K. K. Likharev, Rev. Mod. Phys. 51, 101 (1979)

[19] R. Gati and M. Oberthaler, J. Phys. B 40, R61 (2007).

[20] A. Widera, O. Mandel, M. Greiner, S. Kreim, T. W. Hänsch, and I. Bloch, Phys. Rev. Lett. 92, 160406 (2004)

[21] M. Erhard, H. Schmaljohann, J. Kronjäger, K. Bongs, and K. Sengstock, Phys. Rev. A 69, 032705 (2004).

[22] M. Berry, Czech. J. Phys. 54, 1039 (2004).

[23] W. Heiss, J. Phys. A 45, 444016 (2012).

[24] T. Gao, E. Estrecho, K. Bliokh, T. Liew, M. Fraser, S. Brodbeck, M. Kamp, C. Schneider, S. Höfling, Y. Yamamoto, et al., Nature (London) 526, 554 (2015).

[25] J. Doppler, A. A. Mailybaev, J. Böhm, U. Kuhl, A. Girschik, F. Libisch, T. J. Milburn, P. Rabl, N. Moiseyev, and S. Rotter, arXiv:1603.02325 (2016).

[26] S. A. Gurvitz, Phys. Rev. B 44, 11924 (1991).

[27] S. Gurvitz, Physica Scripta 2015, 014013 (2015).

[28] Y. Meir and N. S. Wingreen, Phys. Rev. Lett. 68, 2512 (1992)

[29] Y. Meir, N. S. Wingreen, and P. A. Lee, Phys. Rev. Lett. 70, 2601 (1993).

[30] M. Bruderer and W. Belzig, Phys. Rev. A 85, 013623 (2012).

[31] G. Schaller, P. Zedler, and T. Brandes, Phys. Rev. A 79, $032110(2009)$
[32] G. E. Topp, T. Brandes, and G. Schaller, EPL (Europhysics Letters) 110, 67003 (2015).

[33] Y. Shin, M. Saba, T. A. Pasquini, W. Ketterle, D. E. Pritchard, and A. E. Leanhardt, Phys. Rev. Lett. 92, 050405 (2004)

[34] H.-J. Lee and R. Bulla, The European Physical Journal B 56, 199 (2007).

[35] H.-J. Lee, K. Byczuk, and R. Bulla, Phys. Rev. B 82, 054516 (2010)

\section{Appendix A: Details of the finite-size simulation}

In the CL, the tunneling elements $t_{k, \alpha}$ and the reservoir frequencies $\omega_{k, \alpha}$ are described simultaneously by the tunnel rates $\Gamma_{\alpha}(\omega)$. For the finite-size simulation we have to separate them again to define the tunneling elements $t_{k, \alpha}$. To this end, we split the tunnel rates into

$$
\Gamma_{\alpha}(\omega)=\Lambda_{\alpha}(\omega) \nu_{\alpha}(\omega)
$$

where $\nu_{\alpha}(\omega) \equiv \rho_{\alpha, 0} \rho(\omega)$ denotes the density of states in the reservoir and $\Lambda_{\alpha}(\omega)=\lambda_{\alpha, 0} \lambda(\omega)$ describes the coupling of the dot and the reservoir modes. In the CL, the density of states diverges, which we achieve formally by $\rho_{\alpha, 0} \rightarrow \infty$, while $\rho(\omega)$ stays constant. Meanwhile, $\lambda_{\alpha, 0} \rightarrow 0$ so that we obtain a finite $\gamma_{\alpha}=\rho_{\alpha, 0} \lambda_{\alpha, 0}$. In the following, we choose $\lambda(\omega)=\left(\omega / \omega_{c}\right)^{\eta}, \nu(\omega)=e^{-\omega / \omega_{c}}$, and $\rho_{\alpha, 0}=k_{\max } / \omega_{c}$.

The frequencies of the reservoir modes are taken as $\omega_{k, \alpha}=-\omega_{c} \log \frac{k_{\max }-k}{k_{\max }}$. In doing so, we make sure that the reconstructed density of states $\nu_{r, \alpha}(\omega)=$ $\sum_{k=0}^{k_{\max }} \delta\left(\omega-\omega_{k, \alpha}\right)$ fullfills

$$
\int_{0}^{\omega_{k, \alpha}} \nu_{r, \alpha}(\omega) d \omega=\int_{0}^{\omega_{k, \alpha}} \nu_{\alpha}(\omega) d \omega .
$$

The tunnel elements $t_{k, \alpha}$ are given by

$$
t_{k, \alpha}^{2}=\Lambda_{\alpha}\left(\omega_{k, \alpha}\right)
$$

for $k>0$. The coupling $t_{0, \alpha}$ is choosen such that

$$
t_{0, \alpha}^{2} \equiv t_{0}^{2} \equiv \Lambda_{\alpha}\left(\frac{1}{\rho_{0}}\right)
$$

as $\Lambda_{\alpha}\left(\omega_{0 \alpha}=0\right)=0$ for $\eta>0$. In doing so, we make sure that the ground state is coupled in the same manner as the excited states close to it. Consequently, for an increasing density of states $\rho_{\alpha, 0}$, the coupling between dot and reservoir ground states decreases.

\section{Appendix B: Rotation of the branch cut}

Here we show, that the modified function $\tilde{C}_{\alpha}(z)$ in Eq. 29 is analytic on the negative imaginary 
axis. For a notational reason we define $R=$ $\{z \in \mathbb{C} \mid \operatorname{Re} z=0 \wedge \operatorname{Im} z<0\}$ which is the negative imaginary axis, and $G=\mathbb{C} \backslash\{z \in \mathbb{C} \mid \operatorname{Re} z<0 \wedge \operatorname{Im} z=0\}$ which is the domain of $\tilde{C}_{\alpha}$. We assume that $C_{\alpha}(z)$ is analytic in the regions $A_{1}$ and $A_{2}$ defined by

$$
\begin{aligned}
& A_{1}=\{z \in G \mid \operatorname{Re} z<0 \wedge \operatorname{Im} z<0\} \\
& A_{2}=G \backslash\left(A_{1} \cup R\right) .
\end{aligned}
$$

Furthermore, we assume, that the analytic continuation of

$$
\Gamma_{\alpha}(i z) \equiv \lim _{\delta \rightarrow 0}\left[C_{\alpha}(z+\delta)-C_{\alpha}(z-\delta)\right]
$$

with $\operatorname{Re} \delta>0$ is analytic for $z \in A_{1} \cup R$. Consequently, $\tilde{C}_{\alpha}(z)$ as defined in 29 is analytic on $A_{1}$ as it is a sum of analytic functions. Additionally, we assume that for all derivatives

$$
C_{\alpha}^{(n)}(z) \equiv \frac{d^{n}}{d z^{n}} C_{\alpha}^{(n)}(z)
$$

with $n \in \mathbb{N}$ the limit

$$
f_{j}^{(n)}(z)=\lim _{\delta \rightarrow 0} C_{\alpha}^{(n)}\left(z+(-1)^{j} \delta\right)
$$

with $j=1,2$ exists for all $z \in R$. Under these requirements, we can now show that $\tilde{C}_{\alpha}(z)$ is indeed analytic for $z \in R$.

To this end, we show that all derivatives $\tilde{C}_{\alpha}^{(n)}(z)$ are continuous for $z \in R$. Therefore, we consider for $z=$ $i \omega \in R$ the limit

$$
\begin{aligned}
\lim _{\delta \rightarrow 0} \tilde{C}_{\alpha}^{(n)} & (-i \omega-\delta) \\
& =\lim _{\delta \rightarrow 0}\left[C_{\alpha}^{(n)}(-i \omega-\delta)+\Gamma_{\alpha}^{(n)}(\omega-i \delta)\right] \\
& =f_{1}^{(n)}(-i \omega)+\Gamma_{\alpha}^{(n)}(\omega)
\end{aligned}
$$

where we have used that $\Gamma_{\alpha}(i z)$ is analytic for $z \in R$. We continue to calculate

$$
\begin{aligned}
\Gamma_{\alpha}^{(n)}(\omega) & \left.\equiv \frac{d^{n}}{d z^{n}} \Gamma_{\alpha}^{(n)}(i z)\right|_{z=-i \omega} \\
& =\frac{d^{n}}{d(-i \omega)^{n}} \lim _{\delta \rightarrow 0}\left[C_{\alpha}(-i \omega+\delta)-C_{\alpha}(-i \omega-\delta)\right] \\
& =\lim _{\delta \rightarrow 0}\left[C_{\alpha}^{(n)}(-i \omega+\delta)-C_{\alpha}^{(n)}(-i \omega-\delta)\right] \\
& =f_{2}^{(n)}(-i \omega)-f_{1}^{(n)}(-i \omega) .
\end{aligned}
$$

Inserting this into Eq. B6 we find

$$
\begin{aligned}
\lim _{\delta \rightarrow 0} \tilde{C}_{\alpha}^{(n)}(-i \omega-\delta) & =f_{2}^{(n)}(-i \omega) \\
& =\lim _{\delta \rightarrow 0} \tilde{C}_{\alpha}^{(n)}(-i \omega+\delta),
\end{aligned}
$$

which proves that $\tilde{C}_{\alpha}(z)$ is analytic on the negative real axis and consequently also analytic on $G$.

\section{Appendix C: Estimation of the branch-cut integral}

Here we derive an estimate for the branch-cut integral corresponding to the term with $k, k^{\prime}=0$ in the second line of Eq. 16). The branch-cut integral $\mathcal{I}_{b c}$ reads

$$
\begin{aligned}
\mathcal{I}_{b c}= & \frac{c_{0, \alpha^{\prime}}}{2 \pi i} \int_{-\infty}^{0} \frac{t_{0, \alpha} t_{0, \alpha^{\prime}} e^{x t}}{\left(x+i \omega_{0, \alpha}\right)\left(x+i \omega_{0, \alpha^{\prime}}\right)} \\
& \times\left[\frac{1}{\tilde{\mathcal{F}}\left(x+i 0^{+}\right)}-\frac{1}{\tilde{\mathcal{F}}\left(x-i 0^{+}\right)}\right] d x .
\end{aligned}
$$

where

$$
\tilde{\mathcal{F}}\left(x \pm i 0^{+}\right)=x+i \epsilon+\sum_{\alpha} \frac{t_{0, \alpha}^{2}}{x+i \omega_{0, \alpha}}+\tilde{C}_{\alpha}\left(x \pm i 0^{+}\right) .
$$

As $t \rightarrow \infty$, the integrand vanishes everywhere in the longtime limit except at $x=0$. Therefore, we investigate the integrand close to that point in the following.

From Eq. 29 we see that

$$
C_{+}(x) \equiv \sum_{\alpha} \tilde{C}_{\alpha}\left(x+i 0^{+}\right)=\sum_{\alpha} \tilde{C}_{\alpha}\left(x-i 0^{+}\right)-\Gamma_{\alpha}(i x) .
$$

Inserting this into C1, we find

$$
\begin{aligned}
\mathcal{I}_{b c}=\frac{c_{0, \alpha^{\prime}}}{2 \pi i} \int_{-\infty}^{0} & \frac{t_{0, \alpha} t_{0, \alpha^{\prime}} \sum_{\alpha} \Gamma_{\alpha}(i x)}{\left(x+i \omega_{0, \alpha}\right)\left(x+i \omega_{0, \alpha^{\prime}}\right)} \\
& \times \frac{e^{x t}}{\tilde{\mathcal{F}}\left(x+i 0^{+}\right) \tilde{\mathcal{F}}\left(x-i 0^{+}\right)} d x .
\end{aligned}
$$

To find the leading contribution of $\mathcal{I}_{b c}$, we have to approximate the terms

$$
\left(x+i \omega_{0, \alpha^{\prime}}\right) \tilde{\mathcal{F}}\left(x \pm i 0^{+}\right)
$$

appearing in the nominator.

To this end, we expand Eq. 22 for $z \approx 0$. First we expand the incomplete Gamma function

$$
\tilde{\Gamma}(-\eta, z)=\tilde{\Gamma}(-\eta)+\frac{z^{-\eta}}{\eta}+z^{-\eta} \mathcal{O}(z) .
$$

Inserting this into Eq. 22 we obtain

$$
\begin{aligned}
C_{+}(z)= & -i \frac{\gamma}{\pi}\left(-i z / \omega_{c}\right)^{\eta} \tilde{\Gamma}(1+\eta) \cdot \tilde{\Gamma}(-\eta) \\
& -i \frac{\gamma}{\pi} \Gamma(\eta)-i \frac{\gamma}{\pi} \tilde{\Gamma}(1+\eta) \mathcal{O}(z) .
\end{aligned}
$$

For $\eta>0$, the second term dominates, which can be identified in this case with the Lamb shift in Eq. 37. For $\eta<0$ the function $C_{+}(z)$ diverges at $z=0$ due to the first term.

\section{Case: $\omega_{0, \alpha}=\omega_{0, \alpha^{\prime}}=0$}

In this case, we find for small $x$

$$
x \tilde{\mathcal{F}}\left(x \pm i 0^{+}\right) \approx \sum_{\alpha=L, R} t_{0, \alpha}^{2}=2 t_{0}^{2},
$$


as we have $\eta>-1$. Here and in the following we assume symmetric tunneling elements $t_{0, L}=t_{0, R} \equiv t_{0}$ for simplicity. We recall that for $\eta<-1$ the integral (19p) diverges for $z=0$. Inserting this into (C4) and using that $\Gamma_{\alpha}(\omega) \approx \gamma_{\alpha}\left(\omega / \omega_{c}\right)^{\eta}$, we obtain

$$
\mathcal{I}_{b c} \approx \frac{c_{0, \alpha^{\prime}}}{2 \pi i} \int_{-\infty}^{0} \frac{\sum_{\alpha} \gamma_{\alpha}}{4 t_{0}^{2}}\left(\frac{-i x}{\omega_{c}}\right)^{\eta} e^{x t} d x .
$$

This integral can be analytically solved. In doing so, we get

$$
\mathcal{I}_{b c} \approx \frac{c_{0, \alpha^{\prime}}}{2 \pi i} \frac{\sum_{\alpha} \gamma_{\alpha}}{4 t_{0}^{2}}\left(\frac{-i}{\omega_{c}}\right)^{\eta} \tilde{\Gamma}(\eta+1) \frac{1}{t^{\eta+1}},
$$

which is expression (31).

\section{Case: $\omega_{0, \alpha^{\prime}} \neq \omega_{0, \alpha}=0$}

In this case, we find

$$
\begin{aligned}
\left(x+i \omega_{0, \alpha^{\prime}}\right) \tilde{\mathcal{F}}\left(x \pm i 0^{+}\right) & \approx \frac{t_{0}^{2} i \omega_{0, \alpha^{\prime}}}{x}, \\
x \tilde{\mathcal{F}}\left(x \pm i 0^{+}\right) & \approx t_{0}^{2},
\end{aligned}
$$

where we have again used that $\eta>-1$. Inserting this into (C4) we get

$$
\begin{aligned}
\mathcal{I}_{b c} & \approx \frac{c_{0, \alpha^{\prime}}}{2 \pi i} \int_{-\infty}^{0} \frac{\sum_{\alpha} \gamma_{\alpha}}{t_{0}^{2} \omega_{0, \alpha^{\prime}}}\left(\frac{-i x}{\omega_{c}}\right)^{\eta}(-i x) e^{x t} d x \\
& =\frac{c_{0, \alpha^{\prime}}}{2 \pi i} \frac{-i \sum_{\alpha} \gamma_{\alpha}}{t_{0}^{2} \omega_{0, \alpha^{\prime}}}\left(\frac{-i}{\omega_{c}}\right)^{\eta} \tilde{\Gamma}(\eta+2) \frac{1}{t^{\eta+2}} .
\end{aligned}
$$

\section{Case: $\omega_{0, \alpha^{\prime}} \neq 0$ and $\omega_{0, \alpha} \neq 0$}

Here we find

$$
\left(x+i \omega_{0, \alpha^{\prime}}\right) \tilde{\mathcal{F}}\left(x \pm i 0^{+}\right) \approx\left\{\begin{array}{ll}
K & \eta>0 \\
K^{\prime} x^{\eta} & \eta<0
\end{array},\right.
$$

where $K, K^{\prime}$ are constants and depend on the system parameters. Inserting this into (C4) we get

$$
\mathcal{I}_{b c} \approx \frac{c_{0, \alpha^{\prime}}}{2 \pi i} \frac{\sum_{\alpha} \gamma_{\alpha}}{K}\left(\frac{-i}{\omega_{c}}\right)^{\eta} \tilde{\Gamma}(\eta+1) \frac{1}{t^{\eta+1}}
$$

for $\eta>0$ and

$$
\mathcal{I}_{b c} \approx \frac{c_{0, \alpha^{\prime}}}{2 \pi i} \frac{\sum_{\alpha} \gamma_{\alpha}}{K^{\prime}}\left(\frac{-i}{\omega_{c}}\right)^{\eta} \tilde{\Gamma}(|\eta|+1) \frac{1}{t^{|\eta|+1}}
$$

for $\eta<0$.

In a similar way, one can show that all other branchcut integrals in Eq. (15) and Eq. (16) vanish even faster as a function of $t$.

\section{Appendix D: Derivation of the roots}

In the following we derive the approximate expression for the location of the roots of $\tilde{\mathcal{F}}(z)$ in Eq. (35). The procedure is performed in two steps. In the first one, we determine the leading order of the imaginary parts which can be used to determine subsequently in the second step the leading order of the real part.

The root which is located at $z=0$ for $t_{0, \alpha} \equiv t_{0}=0$ and $\omega_{0, R}=\omega_{0, L}=0$ is only slightly shifted for a small but finite $t_{0}$. For this reason, we evaluate $\tilde{C}_{\alpha}(z)$ at $z=0$. Assuming additionally $\eta>0$, we thus obtain from

$$
0=\tilde{\mathcal{F}}(z)=z+i \epsilon+\frac{2 t_{0 \alpha}^{2}}{z}+\sum_{\alpha=L, R} \tilde{C}_{\alpha}(z)
$$

the quadratic equation

$$
(z+i \epsilon) z+2 t_{0}^{2}+z i \Sigma(0)=0,
$$

with $\Sigma(0)$ defined by $(33)$. Note that $\Gamma(0)=0$ for $\eta>0$. This equations has the roots

$$
z_{1,2}^{0}=-i \frac{1}{2}\left(\epsilon+\Sigma(0) \pm \sqrt{(\epsilon+\Sigma(0))^{2}+8 t_{0}^{2}}\right) .
$$

We remark that by setting $\Sigma(0)=0$ we obtain the energies of the three-mode system without coupling to the excited reservoir modes. We emphasize that the $z_{j}^{(0)}$ are purely imaginary and thus the leading order of the imaginary part of $z_{j}$.

To determine the leading order of the real parts, we have to determine the next order $z_{j}^{(1)}$ of the roots. To this end, we define

$$
\sum_{\alpha=L, R} \tilde{C}_{\alpha}(z) \equiv i \Sigma(0)+\tilde{C}_{r}(z) .
$$

Inserting $z_{j}=z_{j}^{(0)}+z_{j}^{(1)}$ into $\tilde{\mathcal{F}}(z)=0$ we get

$$
\begin{aligned}
&\left(z_{j}^{(0)}+z_{j}^{(1)}+i \epsilon\right)\left(z_{j}^{(0)}+z_{j}^{(1)}\right)+2 t_{0}^{(2)}+(\mathrm{D} 5) \\
&\left(z_{j}^{(0)}+z_{j}^{(1)}\right)\left[i \Sigma(0)+\tilde{C}_{r}\left(z_{j}^{(0)}+z_{j}^{(1)}\right)\right]=0,
\end{aligned}
$$

which is an exact relation. As before we approximate the argument of $\tilde{C}_{r}\left(z_{j}^{0}+z_{j}^{1}\right) \rightarrow \tilde{C}_{r}\left(z_{j}^{0}\right)$ which is assumed to be small. Furthermore we omit the terms $\left(z_{j}^{1}\right)^{2}$ and $\tilde{C}_{r}\left(z_{j}^{0}\right) z_{j}^{1}$ and arrive at a linear equation with the solution

$$
z_{j}^{1}=\frac{z_{j}^{0} \tilde{C}_{r}\left(z_{j}^{0}\right)}{2 z_{j}^{0}+[i \epsilon+\Sigma(0)]},
$$

We are interested in its real part as we have already identified the leading order of the imaginary part $z_{j}^{(0)}$. Using thus Eq. (33), we finally obtain Eq. (35). 


\section{Appendix E: Derivation of the time-averaged current}

The exact expression for the current reads

$$
\begin{aligned}
I_{R, J}(t) & =-2 \operatorname{Re} i t_{0, R}\left\langle\mathbf{v}_{t, 3}^{\dagger} \mathbf{v}_{t, 1}\right\rangle_{0} \\
& =2 \operatorname{Re} \sum_{j, j^{\prime}} e^{\left(z_{j}^{*}+z_{j^{\prime}}\right) t} \tilde{I}_{R, J}^{C}\left(z_{j}, z_{j^{\prime}}\right), \\
\tilde{I}_{R, J}^{C}\left(z_{j}, z_{j^{\prime}}\right) & \equiv-i t_{0, R} \sum_{l, l^{\prime}} Q_{3, l}^{*}\left(z_{j}\right) Q_{1, l^{\prime}}\left(z_{j^{\prime}}\right)\left\langle\mathbf{v}_{0, l}^{\dagger} \mathbf{v}_{0, l^{\prime}}\right\rangle_{0},
\end{aligned}
$$

where $\mathbf{v}_{t, j}$ is define in Eq. (39). We recall that one root $z_{3}$ of $\tilde{\mathcal{F}}(z)$ converges for the symmetric system $\omega_{0, R} \rightarrow \omega_{0, L}=0$ to $z_{3}=0$. The oscillations are generated by the exponential factor $e^{\left(z_{j}^{*}+z_{j^{\prime}}\right) t}$. We found that $\tilde{I}_{R, J}^{C}\left(z_{j}, z_{j}\right)=0$. The largest period is given by the imaginary part of $z_{3}^{*}+z_{1}$ in regime $I$ and by the imaginary part of $z_{3}^{*}+z_{2}$ in regime II as can be seen in Fig. 3(e). The other root differences are orders of magnitude larger, away from the transition at $\epsilon=\epsilon_{\text {crit }}$. For a notational reason we thus introduce in Eq. (50) the characteristic frequency $\Omega_{J}$.

We define the time-averaged current $\bar{I}_{R, J}(t)$ by neglecting all other frequency contributions in Eqs. (E1). In doing so, the complex current in both regimes reads

$$
\begin{aligned}
& \bar{I}_{R, J}(t)= \\
& \quad-2 \operatorname{Re} i t_{0} e^{-i \Omega_{J} t} \sum_{l, l^{\prime}} Q_{3, l}^{*}(0) Q_{1, l^{\prime}}\left(-i \Omega_{J}\right)\left\langle\mathbf{v}_{0, l}^{\dagger} \mathbf{v}_{0, l^{\prime}}\right\rangle_{0} .
\end{aligned}
$$

To show this, one also has to take into account that

$$
Q\left(z_{3} \rightarrow 0\right)=\left(\begin{array}{ccc}
0 & 0 & 0 \\
0 & \frac{1}{2} & -\frac{1}{2} \\
0 & -\frac{1}{2} & \frac{1}{2}
\end{array}\right)
$$

The matrix elements of $Q_{1,2}\left(-i \Omega_{J}\right)=Q_{1,3}\left(-i \Omega_{J}\right)$ defined in Eq. 44 can be approximated by

$$
\begin{aligned}
Q_{1,2}\left(-i \Omega_{J}\right) & =-\frac{t_{0}}{\Omega_{J}} R_{-i \Omega_{J}}=\frac{t_{0}}{\Omega_{J}} \frac{1}{\left.\frac{d}{d z} \tilde{\mathcal{F}}(z)\right|_{z=-i \Omega_{J}}} \\
& =-\frac{t_{0}}{\Omega_{J}} \frac{1}{1+\frac{2 t_{0}^{2}}{\Omega_{J}^{2}}+\left.\frac{d}{d z} \sum_{\alpha} \tilde{C}_{\alpha}(z)\right|_{z=-i \Omega_{J}}} \\
& \approx-\frac{\Omega_{J}}{2 t_{0}}
\end{aligned}
$$

The approximation is justified as $\Omega_{J}$ is small so that $2 t_{0}^{2} / \Omega_{J}^{2}$ is large compared to the other terms in the nominator. Inserting this into (E3) and using Eqs. (24), we finally obtain Eq. 49. In a similar manner, we also derive the time-averaged dot occupation in Eq. (52).

\section{Appendix F: Josephson current and correlation function}

In the following, we establish a relation between the Josephson current $I_{R, J}(t)$ and the correlation function

$$
\mathcal{C}_{R L}(t) \equiv\left\langle\mathbf{c}_{0, R}^{\dagger} \mathbf{c}_{0, L}\right\rangle_{t},
$$

in order to generalize Eq. (4). However, the relation can not be expressed in a simple way in the time domain as in (4), but has to be done in Fourier space.

Motivated by the theoretical description of electronic systems, we define the complex current operator

$$
\mathbf{I}_{\alpha, J}^{C}=-i t_{0, \alpha} \mathbf{c}_{0, \alpha}^{\dagger} \mathbf{d}
$$

The physical current $I_{R, J}$ is given by $I_{R, J}(t)=$ $2 \operatorname{Re}\left\langle\mathbf{I}_{\alpha, J}^{C}\right\rangle_{t}$. Using E1, we find that the complex current reads

$$
\begin{aligned}
I_{R, J}^{C}(t) & =-i t_{0, R}\left\langle\mathbf{v}_{t, 3}^{\dagger} \mathbf{v}_{t, 1}\right\rangle_{0} \\
& =\sum_{j, j^{\prime}} e^{\left(z_{j}^{*}+z_{j^{\prime}}\right) t} \tilde{I}_{R, J}^{C}\left(z_{j}, z_{j^{\prime}}\right) .
\end{aligned}
$$

The constants $\tilde{I}_{R, J}^{C}\left(z_{j}, z_{j^{\prime}}\right)$ are the Fourier components and are given in Eq. E2. To link the current Fourier components to the correlation function $\mathcal{C}_{R L}(t)$, we express it using its Fourier components

$$
\begin{aligned}
\mathcal{C}_{R L}(t) & =\left\langle\mathbf{v}_{t, 3}^{\dagger} \mathbf{v}_{t, 2}\right\rangle_{0} \\
& =\sum_{j, j^{\prime}} e^{\left(z_{j}^{*}+z_{j^{\prime}}\right) t} \tilde{\mathcal{C}}_{R L}\left(z_{j}, z_{j^{\prime}}\right), \\
\tilde{\mathcal{C}}_{R L}\left(z_{j}, z_{j^{\prime}}\right) & \equiv \sum_{l, l^{\prime}} Q_{3, l}^{*}\left(z_{j}\right) Q_{2, l^{\prime}}\left(z_{j^{\prime}}\right)\left\langle\mathbf{v}_{0, l}^{\dagger} \mathbf{v}_{0, l^{\prime}}\right\rangle_{0} .
\end{aligned}
$$

Now we recognize that

$$
Q_{1, l^{\prime}}\left(z_{j^{\prime}}\right)=-\frac{1}{t_{0, L}}\left(\omega_{0, L}-i z_{j^{\prime}}\right) Q_{2, l^{\prime}}\left(z_{j^{\prime}}\right),
$$

which is obvious from Eq. (44). Inserting this relation in Eq. (E2), we finally obtain

$$
\begin{aligned}
\tilde{I}_{R, J}^{C}\left(z_{j}, z_{j^{\prime}}\right) & =\kappa_{j} \tilde{\mathcal{C}}_{R L}\left(z_{j}, z_{j^{\prime}}\right), \\
\kappa_{j^{\prime}} & =i \frac{t_{0, R}}{t_{0, L}}\left(\omega_{0, L}-i z_{j^{\prime}}\right) .
\end{aligned}
$$

This constitutes an exact relation between the current and the correlation function in Fourier space and therefore generalizes Eq. (4). While the $\tilde{I}_{R, J}^{C}$ and $\tilde{\mathcal{C}}_{R L}$ depend on the initial state, the proportional factor $\kappa_{j}$ depends only on the system parameters. Consequently, the factors $\kappa_{j}$ characterize the current through the transistor as a response to the correlation function. Expressing the current as a function of time, we finally obtain

$$
I_{R, J}(t)=2 \operatorname{Re} \sum_{j, j^{\prime}} e^{\left(z_{j}^{*}+z_{j^{\prime}}\right) t} \kappa_{j^{\prime}} \tilde{\mathcal{C}}_{R L}\left(z_{j}, z_{j^{\prime}}\right) .
$$

